\title{
Onset of oligarchic growth and implication for accretion histories of dwarf planets
}

\author{
Ryuji Morishima $a^{\mathrm{a}, \mathrm{b}, *}$ \\ ${ }^{a}$ University of California, Los Angels, Institute of Geophysics and Planetary Physics, Los Angeles, CA 90095, USA \\ ${ }^{b}$ Jet Propulsion Laboratory/California Institute of Technology, Pasadena, CA 91109, USA
}

\begin{abstract}
We investigate planetary accretion that starts from equal-mass planetesimals using an analytic theory and numerical simulations. We particularly focus on how the planetary mass $M_{\text {oli }}$ at the onset of oligarchic growth depends on the initial mass $m_{0}$ of a planetesimal. Oligarchic growth commences when the velocity dispersion relative to the Hill velocity of the protoplanet takes its minimum. We find that if $m_{0}$ is small enough, this normalized velocity dispersion becomes as low as unity during the intermediate stage between the runaway and oligarchic growth stages. In this case, $M_{\text {oli }}$ is independent of $m_{0}$. If $m_{0}$ is large, on the other hand, oligarchic growth commences directly after runaway growth, and $M_{\text {oli }} \propto m_{0}^{3 / 7}$. The planetary mass $M_{\text {oli }}$ for the solid surface density of the Minimum Mass Solar Nebula is close to the masses of the dwarf planets in a reasonable range of $m_{0}$. This indicates that they are likely to be the largest remnant planetesimals that failed to become planets. The power-law exponent $q$ of the differential mass distribution of remnant planetesimals is typically -2.0 and -2.7 to -2.5 for small and large $m_{0}$. The slope, $q \simeq-2.7$, and the bump at $10^{21} \mathrm{~g}$ (or $50 \mathrm{~km}$ in radius) for the mass distribution of hot Kuiper belt objects are reproduced if $m_{0}$ is the bump mass. On the other hand, small initial planetesimals with $m_{0} \sim 10^{13}$ $\mathrm{g}$ or less are favored to explain the slope of large asteroids, $q \simeq-2.0$, while the bump at $10^{21} \mathrm{~g}$ can be reproduced by introducing a small number of asteroid seeds each with mass of $10^{19} \mathrm{~g}$.
\end{abstract}

Keywords: Accretion; Planetary formation; Origin, Solar System; Asteroids; Kuiper belt

\section{Introduction}

The standard scenario of planet formation begins with small dust floating in a gaseous disk. Clumping of dust particles either due to streaming instability (Johansen et al., 2007, 2015b), turbulent concentration (Cuzzi et al., 2008, 2010; Chambers, 2010; Hopkins, 2016), or direct sticking (Okuzumi et al., 2012; Windmark et al., 2012; Kataoka et al., 2013; Garaud et al., 2013) leads to formation of gravitationally bound planetesimals. These planetesimal formation models generally predict the size of initial planetesimals ranging from 10 to $100 \mathrm{~km}$ or even larger although some direct sticking models (Windmark et al., 2012; Garaud et al., 2013) predict planetesimals as small as $100 \mathrm{~m}$. The initial planetesimal size implied from planetary accretion models is controversial. For models of asteroid formation, Morbidelli et al. (2009) suggested large size $(\sim 100 \mathrm{~km})$ of initial

\footnotetext{
${ }^{*}$ Corresponding author

Email address: Ryuji.Morishima@jpl.nasa.gov (Ryuji Morishima)
} 
planetesimals whereas Weidenschilling (2011) showed that the asteroid size distribution can also be reproduced from initially small $(\sim 100 \mathrm{~m})$ planetesimals. Accretion models for Kuiper belt objects (KBOs) generally favor small initial planetesimals (1-10 km) since the accretion timescale is very long for initially large planetesimals (Kenyon and Bromley, 2012; (Schlichting et al., 2013).

Once planetesimals form, they further grow via mutual collisions. In the early stage of planetary accretion, called the runway growth stage, large planetesimals grow quickly due to their gravitational focusing effects while small planetesimals nearly remain at their initial sizes (Greenberg et al., 1978; Wetherill and Stewart, 1989; Kokubo and Ida, 1996; Barnes et al., 2009). As growth proceeds, the largest bodies, often called planetary embryos or protoplanets, start to dominate the gravitational scattering effect, i.e. viscous stirring (Ida and Makino, 1993). As the number of protoplanets decreases, their orbital separation increases. Eventually, each small body is predominantly stirred by a single protoplanet (Kokubo and Ida, 1998). Because of the viscous stirring effect of each protoplanet, among protoplanets, larger ones grow more slowly than smaller ones. As a result, protoplanets with similar masses grow at a similar rate (Kokubo and Ida, 1998, 2002). This growth mode is called oligarchic growth. Although the basic pictures of runaway and oligarchic growth are apparently well known, the pathway from runaway growth to oligarchic growth described in the literature varies from author to author. The mass of a protoplanet $M_{\text {oli }}$ at the onset of oligarchic growth is not well understood either.

Based on $N$-body simulations and the analytic formulation, Ida and Makino (1993) derived the condition that protoplanets rather than small planetesimals dominate viscous stirring. They argued that once this condition is fulfilled, transition from runaway growth to oligarchic growth occurs. As pointed out by Ormel et al. (2010b), however, this condition is already satisfied even during runaway growth and is thus necessary but not sufficient for oligarchic growth.

Kokubo and Ida (1998) performed direct $N$-body simulations of oligarchic growth and found that the orbital separation of neighboring protoplanets is about 10 Hill radii as a result of orbital repulsion. Since this width is the typical scale of gravitational influence of a protoplanet, this single protoplanet dominates viscous stirring in the region of its gravitational influence. In earlier stages of planetary accretion, the mutual separation between neighboring protoplanets is smaller than 10 Hill radii and their regions of gravitational influence mutually overlap.

Ormel et al. (2010b) derived a new condition for the transition from runaway to oligarchic growth. They examined evolution of the velocity dispersion $u$ of small planetesimals normalized by the Hill velocity $v_{\mathrm{H}}$ of the largest protoplanet, where $v_{\mathrm{H}}$ is the product of Hill radius of the protoplanet and the Keplerian frequency. The normalized velocity $u / v_{\mathrm{H}}$ decreases during the runaway growth while it increases during oligarchic growth. The transition between these two stages then occurs when $u / v_{\mathrm{H}}$ takes its minimum. Based on timescale arguments for the dispersion dominant regime $\left(u / v_{\mathrm{H}}>1\right)$, they derived the planetary mass $M_{\mathrm{oli}}$ at the transition. They showed that $M_{\text {oli }} \propto m_{0}^{3 / 7}$, where $m_{0}$ is the initial mass of each planetesimal. Morishima et al. (2013) derived a similar transition mass without timescale arguments assuming that the protoplanet is the largest body of a continuous mass distribution with the power-law exponent $q$ of -2.5 , which is the typical value during runaway growth (Kokubo and Ida, 1996; Ormel et al., 2010a; (Morishima et al.,, 2013).

Using the simple analytic formulation originally developed by Goldreich et al. (2004), Lithwick (2014) showed that runaway growth is followed by a new stage - the trans-Hill stage - during which $u / v_{\mathrm{H}}$ remains nearly unity. The growth rates of protoplanets during the trans-Hill stage are independent of mass and that leads to $q \simeq-2.0$ for the massive side of the mass distribution. The trans-Hill stage is terminated by emergence of oligarchic growth, in which the mutual separation 
between protoplanets is scaled by the Hill radius (Kokubo and Ida, 1998). The planetary mass $M_{\text {oli }}$ at the onset of oligarchic growth subsequent to the trans-Hill stage is independent of $m_{0}$. The concept of the trans-Hill stage was confirmed by coagulation simulations of Shannon et al. (2015).

Schlichting and Sari (2011) also discussed the transition stage after runaway growth using the analytic formulation of Goldreich et al. (2004). They also found that $u / v_{\mathrm{H}}$ takes a fixed value close to but somewhat larger than unity during the transition stage, contrary to Lithwick (2014). The difference comes from their assumptions; while Schlichting and Sari (2011) assumed that growth of protoplanets is equally contributed by merging with small planetesimals and mutual merging between protoplanets (equal accretion), Lithwick (2014) assumed that growth of protoplanets is dominated by merging with small planetesimals. Lithwick (2014), in fact, analytically proved his assumption and criticized the unjustified assumption employed by Schlichting and Sari (2011).

As reviewed, different authors showed apparently different conditions for or pathways to the onset of oligarchic growth. The objective of the present paper is to establish a comprehensive picture for the onset of oligarchic growth and to derive $M_{\text {oli }}$ for arbitrary $m_{0}$, using an analytic formulation and numerical simulations. Particularly, we will clarify the following three points.

1. Three conditions for the onset of oligarchic growth were indicated in the literature:

(a) protoplanets dominate viscous stirring,

(b) the mutual separation between neighboring protoplanets is 10 Hill radii, and

(c) $u / v_{\mathrm{H}}$ takes its minimum.

The condition (b) is equivalent to the definition of oligarchic growth. We will show that the condition (c) is useful to pin down the timing of the onset of oligarchic growth, as shown by Ormel et al. (2010b), although the condition (a) is also required for oligarchic growth.

2. Ormel et al. (2010b) and Morishima et al. (2013) showed that both $M_{\text {oli }}$ and the minimum $u / v_{\mathrm{H}}$ depend on $m_{0}$ while Schlichting and Sari (2011) and Lithwick (2014) showed that $M_{\mathrm{oli}}$ and the minimum $u / v_{\mathrm{H}}$ are both independent of $m_{0}$. We will show that the formulations of Ormel et al. (2010b) and Morishima et al. (2013) are applicable for large $m_{0}$ while the formulations of Schlichting and Sari (2011) and Lithwick (2014) are applicable for small $m_{0}$. The critical value of $m_{0}$ which separates these two regimes will be explicitly formulated. The transition stage between the runway growth and oligarchic growth stages appears only if $m_{0}$ is lower than this critical value.

3. Schlichting and Sari (2011) and Lithwick (2014) employed different assumptions for the contribution of mutual merging between protoplanets to their growth. We will show that as long as their equations are employed, growth of protoplanets is dominated by merging with small planetesimals, as proved by Lithwick (2014). We will, however, show that the contribution of mutual merging can be comparable to or even larger than the small bodies' contribution due to very small inclinations of large bodies and to small bodies' velocities somewhat larger than the Hill velocity of large bodies.

Besides clarifying the picture of the onset of oligarchic growth, at least, the following two important implications are discussed from $M_{\text {oli }}$. First, the masses of the dwarf planets are potentially close to $M_{\text {oli }}$. Protoplanets more massive than $M_{\text {oli }}$ have low velocity dispersion due to dynamical friction of surrounding planetesimals. As a result, they efficiently merge together and form large planets, while leaving remnant planetesimals with the largest one about $M_{\text {oli }}$ in mass (Morishima et al., 2008). This argument is probably applied to asteroids. For KBOs at large heliocentric distances, planetary accretion is likely to be incomplete. Recent observations of KBOs (Fraser et al., 2014; Adams et al., 2014) showed that the largest two bodies, Pluto and Eris, are 
distinctively separated from a continuous size distribution, possibly indicating that they actually entered into oligarchic growth but their growth was stalled immediately after that. In either case, it is worthwhile to compare the masses of the dwarf planets in the asteroid and Kuiper belts with $M_{\text {oli }}$ as it helps us to infer the physical parameters of the proto-solar nebula. In 2015, NASA's Dawn and New Horizons spacecraft arrived at Ceres (Russell et al., 2015) and Pluto (Stern et al., 2015), respectively. The present study may be able to give a hint for why they failed to become planets. Additional constraints on their accretion histories are obtained from the mass distribution slopes of asteroids and KBOs.

Second, rapid migration of protoplanets caused by gravitational interactions with surrounding planetesimals is likely to occur once their masses reach $\sim M_{\text {oli }}$. A protoplanet gravitationally scatters surrounding planetesimals. Back reaction on the protoplanet causes its radial migration, called planetesimal-driven migration (PDM), as the exerted torques do not cancel out (Ida et al., 2000; Kirsh et al., 2009; Bromley and Kenvon, 2011; Ormel et al., 2012; Kominami et al., 2016). $\mathrm{PDM}$ is important for planet formation as it can significantly modify planetary growth rates and mutual separations between planets (Levison et al., 2010). Minton and Levison (2014) pointed out that the protoplanet must be much more massive than other nearby bodies to trigger smooth PDM. Otherwise, stochastic forces from nearby bodies prohibit smooth PDM. They found that such a condition is generally fulfilled at large distance from the central star ( $>1 \mathrm{AU})$ and with the protoplanet's mass of about the lunar mass, although the initial planetesimal size was limited to $\sim 100 \mathrm{~km}$ in their study. The condition of mass separation is expected to be fulfilled at best at the onset of oligarchic growth, since the largest protoplanet is caught up with by other protoplanets in mass in the midst of oligarchic growth. Particle-based hybrid codes (Levison et al., 2012; Morishima, 2015) for planetary accretion developed recently can handle PDM of protoplanets. The lowest protoplanet's mass capable in these codes is the mass of a tracer, which represents a large number of planetesimals. Thus, $M_{\text {oli }}$ is a good indicator for the tracer mass that should be used in these new codes.

In Section 2, we develop an analytic theory for planetary growth and derive the planetary mass $M_{\text {oli }}$ at the onset of oligarchic growth for arbitrary initial mass of each planetesimal $m_{0}$. In Section 3, we perform numerical simulations to check the validity of our theory using a pure $N$-body code (Morishima et al., 2010) and a particle-based hybrid code (Morishima, 2015). Implications of the present study and effects not considered in Sections 2 and 3 are discussed in Section 4. The summary is given in Section 5 .

\section{Theory}

We employ the two-component model developed by Goldreich et al. (2004) and use their notation as much as possible. Consider a system of small and large bodies with the surface densities of $\sigma$ and $\Sigma$, respectively. The mass, radius, and velocity dispersion of the small bodies are $m_{0}, s$, and $u$ and those for large bodies are $M, R$, and $v$. All the bodies have the bulk density $\rho$. This two component approximation is easily extended to a continuous size distribution case.

The Hill radius of a large body is

$$
R_{\mathrm{H}}=a h_{M}=a\left(\frac{M}{3 M_{\odot}}\right)^{1 / 3}
$$

where $a$ is the semimajor axis of the body, $h_{M}$ is the reduced Hill radius, and $M_{\odot}$ is the mass of the central star which we assume to be the solar mass. The ratio of the physical radius to the Hill 
radius is defined as

$$
\alpha=\frac{R}{R_{\mathrm{H}}}=6.0 \times 10^{-3}\left(\frac{a}{1 \mathrm{AU}}\right)^{-1}\left(\frac{\rho}{2 \mathrm{~g} \mathrm{~cm}^{-3}}\right)^{-1 / 3} .
$$

The Hill velocity of the large body is given as

$$
v_{\mathrm{H}}=R_{\mathrm{H}} \Omega,
$$

where $\Omega$ is the Keplerian frequency at $a$.

The change rate of the velocity dispersion $u$ due to large bodies' viscous stirring is

$$
\frac{1}{u} \frac{\mathrm{d} u}{\mathrm{~d} t}=\frac{\Sigma \Omega}{\rho R} \alpha^{-2} \begin{cases}\left(v_{\mathrm{H}} / u\right)^{4} & \left(\text { if } u>v_{\mathrm{H}}\right), \\ v_{\mathrm{H}} / u & \left(\text { if } u<v_{\mathrm{H}}\right) .\end{cases}
$$

Here we ignored collisional damping and gas drag. These effects are generally unimportant unless $s$ is very small (see Section 4.5).

For a more general continuous size distribution, the change rate of $u$ due to all the bodies' viscous stirring is obtained if we replace $\Sigma$ by $M_{\text {stir }} \sigma / M$ in Eq. (4). Here $M_{\text {stir }}$ is the effective mass for viscous stirring (Ida and Makino, 1993; Ormel et al., 2010b) given as

$$
M_{\text {stir }}=\frac{\left\langle m^{2}\right\rangle}{\langle m\rangle}=-\frac{q+2}{q+3}\left(\frac{M}{m_{0}}\right)^{q+2} M,
$$

where the brackets represent averaging over mass $m$ between $m_{0}$ and $M$. The latter equivalence in Eq. (5) is applied if the mass distribution is given by a single power-law distribution $d N_{c}=m^{q} d m$, where $N_{c}$ is the cumulative number of the bodies in the system and $q$ is the power-law exponent. We also assumed that $-3<q<-2$. This range of $q$ appears most commonly in planetary accretion around the onset of oligarchic growth, although $M_{\text {stir }}$ for other ranges of $q$ can be derived easily. If $q>-3$, large bodies dominate viscous stirring (Ida and Makino, 1993). This is expressed as

$$
M \Sigma \simeq M_{\mathrm{stir}} \sigma,
$$

and we recover Eq. (4).

\subsection{Growth due to merging with small bodies}

We first consider growth of large bodies due to merging with small bodies. The contribution of mutual merging between large bodies is discussed in the next section. The growth rate of the large body is

$$
\frac{1}{R} \frac{\mathrm{d} R}{\mathrm{~d} t}=\frac{\sigma \Omega}{\rho R} \alpha^{-1} \begin{cases}\left(v_{\mathrm{H}} / u\right)^{2} & \left(\text { if } v_{\mathrm{H}}<u<\alpha^{-1 / 2} v_{\mathrm{H}}\right), \\ v_{\mathrm{H}} / u & \left(\text { if } \alpha^{1 / 2} v_{\mathrm{H}}<u<v_{\mathrm{H}}\right),\end{cases}
$$

where $\alpha^{-1 / 2} v_{\mathrm{H}}$ is about the escape velocity of the largest body.

The ratio $\Sigma / \sigma$ is called the efficiency (Schlichting and Sari, 2011; Lithwick, 2014). Provided that the normalized velocity dispersion, $u / u_{\mathrm{H}}$, evolves quasi-stationary, the relationship between the efficiency $\Sigma / \sigma$ and the velocity dispersion $u / v_{\mathrm{H}}$ at a certain time is derived by adopting $\mathrm{d}\left(u / v_{\mathrm{H}}\right) / \mathrm{d} t=0$ or equivalently by equating Eq. (4) with Eq. (7) as

$$
\frac{\Sigma}{\sigma}=\alpha\left(\frac{u}{v_{\mathrm{H}}}\right)^{2} .
$$

As we will describe in detail below, planetary accretion is classified into three distinct stages. 
1. Runaway growth: $\mathrm{d}(\Sigma / \sigma) / \mathrm{d} M<0, q<-2.0$, and $\mathrm{d}(\dot{R} / R) / \mathrm{d} R>0$.

2. Trans-Hill (neutral): $\mathrm{d}(\Sigma / \sigma) / \mathrm{d} M=0, q=-2.0$, and $\mathrm{d}(\dot{R} / R) / \mathrm{d} R=0$.

3. Oligarchic growth: $\mathrm{d}(\Sigma / \sigma) / \mathrm{d} M>0, q>-2.0$, and $\mathrm{d}(\dot{R} / R) / \mathrm{d} R<0$.

Here the index $q$ is used for largest bodies only.

During the runaway growth, $\Sigma / \sigma\left(\simeq M_{\text {stir }} / M\right)$ and $u / v_{\mathrm{H}}$ decrease with increasing $M$, provided that $q$ changes only slowly (Eq. (5)). Typically, $q \simeq-2.7$ to -2.5 (Kokubo and Ida, 1996; Ormel et al., 2010b). In other words, $v_{\mathrm{H}}$ (or $R$ ) grows faster than $u$ does. Once the ratio $u / v_{\mathrm{H}}$ becomes as low as unity, the change rates of $u$ and $v_{\mathrm{H}}$ (or $R$ ) become the same. As a result, $u / v_{\mathrm{H}}$ is kept to be nearly unity. The growth rates of large bodies are independent of mass (neutral growth) as long as their Hill velocities are larger than the velocity dispersion. Thus, this stage, named the trans-Hill stage (Lithwick, 2014), is technically different from the traditional runaway growth stage, in which larger bodies grow faster. During this stage, $q$ is expected to increase toward -2 as indicated by a constant $\Sigma / \sigma$ and neutral growth. The trans-Hill stage is terminated by emergence of oligarchic growth.

The above discussion for the trans-Hill stage is applied only if $m_{0}$ is small enough. For large $m_{0}$, oligarchic growth commences directly after runaway growth before $u / v_{\mathrm{H}}$ reaches to unity in the absence of the trans-Hill stage, as shown below.

Oligarchic bodies gravitationally repulse each other and their mutual distance is kept to be about $b_{e}$ times the mutual Hill radii, where $b_{e} \sim 10$ (Kokubo and Ida, 1998). At the end of oligarchic growth, all the nearby planetesimals are accreted by protoplanets. The final mass of an oligarchic body is called the isolation mass $M_{\text {iso }}$ (Kokubo and Ida, 2002) given as

$$
M_{\text {iso }}=2 \pi b_{e} a\left(2^{1 / 3} R_{\mathrm{H}}\right) \sigma=\left(2 \pi b_{e} a^{2} \sigma\right)^{3 / 2}\left(\frac{2}{3 M_{\odot}}\right)^{1 / 2},
$$

where $2^{1 / 3} R_{\mathrm{H}}$ is the mutual Hill radius and $\sigma$ is the value at the beginning. Using $M_{\text {iso }}$ and Eq. (8), the mass of the oligarchic body in the midst of oligarchic growth is given as

$$
M=\left(\frac{\Sigma}{\sigma}\right)^{3 / 2} M_{\mathrm{iso}}=\alpha^{3 / 2}\left(\frac{u}{v_{\mathrm{H}}}\right)^{3}(q+3)^{3 / 2} M_{\mathrm{iso}},
$$

where the effect of depletion of small bodies is ignored.

If $m_{0}$ is small enough, the largest body's mass at the transition from trans-Hill to oligarchic growth is given as

$$
M_{\mathrm{oli}, \mathrm{SS}}=\alpha^{3 / 2} M_{\mathrm{iso}},
$$

as $u / v_{\mathrm{H}}=1$ and $q \simeq-2$. The same transition mass except for the factor was derived by Lithwick (2014). The efficiency at the transition for small $m_{0}$ is

$$
\left(\frac{\Sigma}{\sigma}\right)_{\mathrm{SS}}=\alpha .
$$

If $m_{0}$ is not small enough, the largest body's mass at the transition from runaway to oligarchic growth is given as

$$
M_{\mathrm{oli}, \mathrm{L}}(q)=\left[\left(-\frac{q+2}{q+3}\right)^{3} m_{0}^{-3(q+2)} M_{\mathrm{iso}}^{2}\right]^{-1 /(3 q+4)},
$$


where we used Eqs. (5), (6), and (10). If $q=-2.5$, we have

$$
M_{\text {oli,L }}(q=-2.5)=m_{0}^{3 / 7} M_{\text {iso }}^{4 / 7} .
$$

The same equation except for the factor was derived by Morishima et al. (2013). The transition mass derived by Ormel et al. (2010b) is also similar to but slightly different from Eq. (14) since they employed different assumptions. The efficiency and the lowest $u / v_{\mathrm{H}}$ at the transition for large $m_{0}$ are respectively given as

$$
\left(\frac{\Sigma}{\sigma}\right)_{\mathrm{L}}=\left(-\frac{q+3}{q+2}\right)^{2}\left(\frac{m_{0}}{M_{\mathrm{iso}}}\right)^{2(q+2) /(3 q+4)},
$$

and

$$
\left(\frac{u}{v_{\mathrm{H}}}\right)_{\mathrm{L}}=\alpha^{-1 / 2}\left(\frac{\Sigma}{\sigma}\right)_{\mathrm{L}}^{1 / 2}
$$

The critical planetesimal mass that separates these two regimes is given by equating Eq. (11) with Eq. (14) as $\alpha^{7 / 2} M_{\text {iso }}$ for $q=-2.5$.

\subsection{Corrections due to merging between large bodies}

Now we consider the contribution of merging between large bodies to their growth. Provided that $u \geq v_{\mathrm{H}}$, the accretion rate of large bodies is given as

$$
\frac{1}{R} \frac{\mathrm{d} R}{\mathrm{~d} t}=\frac{\sigma \Omega}{\rho R} \alpha^{-1}\left(\frac{v_{\mathrm{H}}}{u}\right)^{2}+\frac{\Sigma \Omega}{\rho R} \alpha^{-1} \begin{cases}\left(v_{\mathrm{H}} / v\right)^{2} & \text { (if } \left.v_{\mathrm{H}}<v<\alpha^{-1 / 2} v_{\mathrm{H}}\right), \\ v_{\mathrm{H}} / v & \text { (if } \left.\alpha^{1 / 2} v_{\mathrm{H}}<v<v_{\mathrm{H}}\right), \\ \alpha^{-1 / 2} & \left(\text { if } v<\alpha^{1 / 2} v_{\mathrm{H}}\right),\end{cases}
$$

where the first and the second terms in the r.h.s are the contributions from merging with small and large bodies, respectively. The large bodies' velocity $v$ is determined by the balance between mutual stirring and dynamical friction due to small bodies:

$$
\frac{1}{v} \frac{\mathrm{d} v}{\mathrm{~d} t}=-\frac{\sigma \Omega}{\rho R} \alpha^{-2}\left(\frac{v_{\mathrm{H}}}{u}\right)^{4}+\frac{\Sigma \Omega}{\rho R} \alpha^{-2} \begin{cases}\left(v_{\mathrm{H}} / v\right)^{4} & \left(\text { if } v_{\mathrm{H}}<v\right), \\ v_{\mathrm{H}} / v & \left(\text { if } v<v_{\mathrm{H}}\right) .\end{cases}
$$

The velocity $v$ at the equilibrium state $(\mathrm{d} v / \mathrm{d} t=0)$ is

$$
\frac{v}{v_{\mathrm{H}}}= \begin{cases}\alpha^{1 / 4}\left(u / v_{\mathrm{H}}\right)^{3 / 2} & \left(\text { if } v_{\mathrm{H}}<v\right) \\ \alpha\left(u / v_{\mathrm{H}}\right)^{6} & \left(\text { if } v<v_{\mathrm{H}}\right)\end{cases}
$$

where we used Eq. (8). The ratio of the large bodies' contribution to the small bodies' contribution is then given as

$$
\frac{\mathrm{d} R /\left.\mathrm{d} t\right|_{\text {large }}}{\mathrm{d} R /\left.\mathrm{d} t\right|_{\text {small }}}= \begin{cases}1 & \left(\text { if } v=u=\alpha^{-1 / 2} v_{\mathrm{H}}\right), \\ \alpha^{1 / 3} & \left(\text { if } v=v_{\mathrm{H}} \text { and } u=\alpha^{-1 / 6} v_{\mathrm{H}}\right), \\ \alpha^{1 / 6} & \left(\text { if } v=\alpha^{1 / 2} v_{\mathrm{H}} \text { and } u=\alpha^{-1 / 12} v_{\mathrm{H}}\right), \\ \alpha^{1 / 2} & \left(\text { if } v<\alpha^{1 / 2} v_{\mathrm{H}} \text { and } u=v_{\mathrm{H}}\right) .\end{cases}
$$

Since $\alpha<1$, the contribution of large bodies is always lower than that of small bodies. Equal accretion assumed by Schlichting and Sari (2011) is not justified, as long as Eqs. (17) and (18) are employed. 
It is not necessary to distinguish between the horizontal and vertical velocities in Eqs. (17) and (18) in the dispersion dominant regime $\left(v / v_{\mathrm{H}}>1\right)$. However, their difference plays a crucial role in the shear dispersion regime $\left(v / v_{\mathrm{H}}<1\right)$. The accretion rate in the shear dominant regime only depends on the vertical velocity $v_{\perp}$ (e.g., Ida and Nakazawa, 1989). Thus, $v$ is replaced by $v_{\perp}$ if $v<v_{\mathrm{H}}$ in Eqs. (17). The viscous stirring rate of $v_{\perp}$ in the shear dominant regime is smaller by $v_{\perp} / v$ than that given by Eq. (18) (Goldreich et al., 2004, their Section 4.4). This means that if $v<v_{\mathrm{H}}, v_{\perp}$ evolves toward zero as dynamical friction becomes always larger than vertical stirring. Thus, we may be able to apply the highest possible value for the second term of the r.h.s of Eq. (17) as long as $v<v_{\mathrm{H}}$ or $u<\alpha^{-1 / 6} v_{\mathrm{H}}$. After taking into account this effect, the relative contribution of large bodies turns out as

$$
\frac{\mathrm{d} R /\left.\mathrm{d} t\right|_{\text {large,correct }}}{\mathrm{d} R /\left.\mathrm{d} t\right|_{\text {small }}}= \begin{cases}\alpha^{-1 / 6} & \left(\text { if } u=\alpha^{-1 / 6} v_{\mathrm{H}}\right), \\ 1 & \left(\text { if } u=\alpha^{-1 / 8} v_{\mathrm{H}}\right), \\ \alpha^{1 / 2} & \left(\text { if } u=v_{\mathrm{H}}\right) .\end{cases}
$$

In the rage of $\alpha^{-1 / 8}<u / v_{\mathrm{H}}<\alpha^{-1 / 6}$, the large bodies' contribution becomes large than the small bodies' contribution.

If growth of large bodies is dominated by mutual merging, the velocity dispersion $u / v_{\mathrm{H}}$ may be determined by equating Eq. (4) with the second term of the r.h.s of Eq. (17) instead of the first term used in Eq. (8). This gives

$$
\left(\frac{u}{v_{\mathrm{H}}}\right)_{\mathrm{S}}=\alpha^{-1 / 8}
$$

at which the contributions from large and small bodies are equal (equal accretion). The question is whether this state can remain avoiding $u / v_{\mathrm{H}}$ from further decrease down to unity where the contribution of small bodies dominates. We infer that the factors of order of unity ignored so far in the stirring and accretion rates are important. Let us consider a factor somewhat larger than unity in front of the second term of Eq. (17) for $v<\alpha^{1 / 2} v_{\mathrm{H}}$. For simplicity, the same factor is applied to Eq. (4). The detailed numerical calculations of the accretion rates (Ida and Nakazawa, 1989; Greenzweig and Lissauer, 1990) and the stirring rates (Ida, 1990) support this idea. Then, the big bodies' contribution still dominates even at the equilibrium velocity, $u=\alpha^{-1 / 8} v_{\mathrm{H}}$, while the small bodies' contribution takes a fixed value relative to the large bodies' one. This continues even at a slightly lower $u$ while the viscous stirring timescale becomes shorter than the accretion timescale. As a result, $u / v_{\mathrm{H}}$ increases towards the equilibrium value again. We also call this stage after runaway growth, as the trans-Hill stage, although $u / v_{\mathrm{H}}$ does not exactly go down to unity.

The planetary mass at the beginning of the trans-Hill stage with $u / v_{\mathrm{H}}=\alpha^{-1 / 8}$ is given as

$$
M=M_{\operatorname{tran}}(q)=\left[-\frac{(q+3)^{2}}{q+2}\right]^{1 /(q+2)} \alpha^{3 /[4(q+2)]} m_{0} .
$$

For $q=-2.5, M_{\text {tran }}=4 \alpha^{-3 / 2} m_{0}$. The efficiency during the trans-Hill stage is given as

$$
\left(\frac{\Sigma}{\sigma}\right)_{\mathrm{S}}=f_{\mathrm{s}} \alpha^{3 / 4}
$$

where $f_{\mathrm{s}}$ is the order of unity factor, which we estimate to be 0.5 from our simulations. This equation is the same as the one derived by Schlichting and Sari (2011). Note that, however, they derived this efficiency assuming equal accretion without justification while we interpret this 
efficiency realized as an effect of large-body dominant accretion. Since the efficiency takes a fixed value independently of $M$, the mass distribution for large bodies is expected to have $q=-2$ also in this case (but see the discussion below). Inserting Eq. (24) into Eq. (10), the planetary mass at the onset of oligarchic growth is

$$
M_{\mathrm{oli}, \mathrm{S}}=f_{\mathrm{s}}^{3 / 2} \alpha^{9 / 8} M_{\text {iso }} .
$$

To summarize, the embryo mass $M_{\mathrm{oli}}$ and the velocity dispersion $\left(u / v_{\mathrm{H}}\right)_{\mathrm{oli}}$ at the onset of oligarchic growth are

$$
M_{\mathrm{oli}}= \begin{cases}M_{\mathrm{oli}, \mathrm{S}} & \left(\text { if } m_{0}<m_{0, \mathrm{crit}}\right), \\ M_{\mathrm{oli}, \mathrm{L}} & \left(\text { if } m_{0}>m_{0, \mathrm{crit}}\right)\end{cases}
$$

and

$$
\left(\frac{u}{v_{\mathrm{H}}}\right)_{\text {oli }}= \begin{cases}\left(u / v_{\mathrm{H}}\right)_{\mathrm{S}} & \left(\text { if } m_{0}<m_{0, \text { crit }}\right), \\ \left(u / v_{\mathrm{H}}\right)_{\mathrm{L}} & \left(\text { if } m_{0}>m_{0, \text { crit }}\right),\end{cases}
$$

where the critical planetesimal mass $m_{0, \text { crit }}$ that separates these two regimes is given by equating Eq. (25) with Eq. (14) as

$$
m_{0, \text { crit }}=f_{\mathrm{s}}^{7 / 2} \alpha^{21 / 8} M_{\text {iso }} .
$$

We use $M_{\mathrm{oli}, \mathrm{S}}$ as the prediction of the planetary mass at the onset of oligarchic growth for small $m_{0}$, although its difference from $M_{\text {oli,SS }}$ [Eq. (11)] is quite small.

The argument here has some unresolved issues. First, the accretion of large bodies with $v<$ $\alpha^{1 / 2} v_{\mathrm{H}}$ is not neutral but ordered $(\mathrm{d}(\dot{R} / R) / \mathrm{d} R<0)$. All large bodies in the ordered regime eventually converge in mass and this leads to increase of $\Sigma / \sigma$, whereas we see a constant $\Sigma / \sigma$ or $q=-2$ in our simulations for small $m_{0}$. Since the mass range of large bodies in the ordered regime is generally very narrow ( or $v_{\perp} \sim \alpha^{1 / 2} v_{\mathrm{H}}$ ) in simulations, this effect seems limited. Second, the solution of $u=v_{\mathrm{H}}$ (Section 2.1) is not excluded by the theory. The solution of $u=\alpha^{-1 / 8} v_{\mathrm{H}}$ is realized in our simulations probably because this solution comes earlier than the one with $u=v_{\mathrm{H}}$. However, it is unclear whether the former one is preferentially realized in arbitrary initial conditions such as one starting with $u=v_{\mathrm{H}}$ and $\Sigma / \sigma \sim \alpha$.

We have discussed the contribution of large bodies during the trans-Hill stage. To complete the section, we discuss their contribution during the runway and oligarchic growth stages. The large bodies' contribution during oligarchic growth is estimated to be half of the small bodies' contribution if $b_{e}$ is fixed (Chambers, 2006). The large bodies' contribution can be larger if $b_{e}$ increases with time (Morishima et al., 2013). If mutual merging between protoplanets is prohibited in oligarchic growth, their influences of gravity eventually overlap $\left(b_{e}\right.$ decreases) and this violates the definition of oligarchic growth. Therefore, equal accretion or large-body dominant accretion is actually a necessary condition for the onset of oligarchic growth, consistent with the correction given in this subsection.

The argument for the large bodies' contribution during runway growth is complicated. For $v>v_{\mathrm{H}}$, the theory predicts that the contribution of small bodies is dominant [Eq. [20)]. However, numerical simulations (Ormel et al., 2010a, see also Section 3) show that the contribution of large bodies is comparable to that of small bodies even during the runway growth stage and different interpretations than those for the trans-Hill stage are necessary. We attribute the discrepancy to an overestimation of $v$ in the analytic model. If Eq. (18) is applied to a continuous size distribution, one can find that $v^{\prime} \propto m^{-1 / 4}$ for $M_{\text {stir }}<m<M$, where $v^{\prime}$ is the velocity dispersion of bodies with mass $m$, while $v^{\prime}$ is constant $(=u)$ for $m<M_{\text {stir }}$ (Goldreich et al., 2004). On the other hand, simulations of runaway growth show $v^{\prime} \propto m^{-1 / 4}$ down to the smallest bodies while the mass distribution has 
$q \simeq-2.5$ (Morishima et al., 2008). It is likely that the velocity distribution does not fully evolve to the equilibrium state as the growth timescale of large bodies is shorter (see also Rafikov, 2003). Taking this effect into account, one can find that large and small bodies contribute almost equally to growth of large bodies during runaway growth $\left(\mathrm{d} \dot{M} / \mathrm{d} \log m_{\mathrm{imp}} \propto m_{\mathrm{imp}}^{q+2-2 \beta} \sim\right.$ const, where $m_{\mathrm{imp}}$ is the impactor's mass and $\beta=\mathrm{d} \log v^{\prime} / \mathrm{d} \log m_{\mathrm{imp}}$, provided that all bodies are in the dispersion dominant regime). The combination of the values of $\beta \simeq-1 / 4$ and $q \simeq-2.5$ is also consistent with the steady-state mass distribution, $q=-13 / 6+\beta$ (Makino et al., 1998). The argument here does not modify $M_{\mathrm{oli}, \mathrm{L}}[\mathrm{Eq}$. (14)], as the derivation was simply based on the assumption of $q=-2.5$.

\section{Numerical simulations}

\subsection{Simulation codes}

We simulate planetary accretion that starts from equal-mass planetesimals each with the mass of $m_{0}$, using two different codes. If $m_{0}$ is large and the initial number of planetesimals $N$ is not very large, we perform pure $N$-body simulations using the parallel-tree code, pkdgrav2 (Morishima et al., 2010). The gravitational solver employed by the code is a parallelized tree method, in which the gravitational forces from distant particles are calculated using the multipole expansions (Richardson et al., 2000; Stadel, 2001). The computational cost for the gravity calculation is proportional to only $N \log N$ with tree methods. The code employs the symplectic integrator, SyMBA (Duncan et al., 1998), that handles close encounters between planetesimals using multi time-stepping. The combination of the advanced gravity solver and the integrator allows us to handle a large number of particles while keeping a large time step. Unfortunately, pure $N$-body simulations are still computationally intense and cannot practically handle $N$ more than $\sim 10^{4}$, provided that a number of time steps is hundreds of millions for a simulation of oligarchic growth.

To overcome such a situation, we recently have developed a particle-based hybrid code (Morishima, 2015). We use this new code if $m_{0}$ is small and $N$ is very large. The code retains various advantages of direct $N$-body calculations as the gravitational accelerations due to planetary embryos are calculated by the $N$-body routine. The code can also handle a large number of small planetesimals using the super-particle approximation, in which a large number of small planetesimals are represented by a small number of tracers. Collisional and gravitational interactions between tracers are handled by a statistical routine that uses the phase-averaged collision and stirring rates. As planetesimals grow, the number of planetesimals in a tracer decreases. Once the number of planetesimals in a tracer becomes unity, this particle is promoted to a sub-embryo. After the sub-embryo becomes massive enough, it is further promoted to a full-embryo. The acceleration of the sub-embryo due to surrounding planetesimals is handled by the statistical routine to avoid artificially strong kicks on the sub-embryo while the acceleration of the full-embryo is always handled by the $N$-body routine. The algorithms and various tests for the validation of the code are described in detail in Morishima (2015).

In a hybrid simulation, the tracer mass $m_{t 0}$, which is inversely proportional to the initial number of tracers $N_{\text {tr }}$, needs to be chosen. Since $m_{t 0}$ is also a minimum mass of a sub-embryo handled by the code, it is desirable that $m_{t 0}$ is lower than $M_{\text {oli }}[\mathrm{Eq} .(26)]$ to examine the onset of oligarchic growth in detail. This condition is roughly fulfilled in all the simulations (Table 1). 


\begin{tabular}{|cccc|}
\hline Region (AU) & $0.09-0.11$ & $0.9-1.1$ & $27-33$ \\
$f_{\sigma}$ & 1 & 1 & 10 \\
$M_{\text {total }}(\mathrm{g})$ & $6.3 \times 10^{26}$ & $2.0 \times 10^{27}$ & $4.6 \times 10^{29}$ \\
$M_{\text {iso }}(\mathrm{g})$ & $1.0 \times 10^{26}$ & $5.8 \times 10^{26}$ & $2.0 \times 10^{30}$ \\
$\delta t$ (days) & 0.2 & 6 & 3600 \\
$m_{0}$ (Hybrid) (g) & \multicolumn{3}{c}{$10^{13}, 10^{17}, 10^{21}$} \\
$m_{0}(N$-body) $(\mathrm{g})$ & $3.1 \times 10^{23}$ & $10^{24}$ & $\mathrm{~N} / \mathrm{A}$ \\
$m_{t 0}(\mathrm{~g})$ & $6.3 \times 10^{22}$ & $2.0 \times 10^{23}$ & $4.6 \times 10^{25}$ \\
$M_{\text {oli, }}(\mathrm{g})$ & $1.5 \times 10^{24}$ & $6.4 \times 10^{23}$ & $4.9 \times 10^{25}$ \\
\hline
\end{tabular}

Table 1. Parameters used for simulations. Region represents the initial inner and outer edges of the planetesimal annulus. $f_{\sigma}$ is the scaling factor for the solid surface density relative to MMSN. $M_{\text {total }}$ is the initial total mass of planetesimals. $M_{\text {iso }}$ is the isolation mass for $b_{\mathrm{e}}=10$ [Eq. (9)]. $\delta t$ is the time step for orbital integration. $m_{0}$ is the initial mass of a planetesimal. $m_{t 0}$ is the initial mass of a tracer for hybrid simulations with $N_{\mathrm{tr}}=10^{4} . M_{\mathrm{oli}, \mathrm{S}}$ is the predicted embryo mass at the onset of oligarchic growth for small $m_{0}$ [Eq. (25)] .

\subsection{Initial conditions and collisional outcome}

The initial surface density of planetesimals is given by

$$
\sigma=f_{\sigma} \sigma_{\mathrm{M} 1}\left(\frac{a}{1 \mathrm{AU}}\right)^{-1.5}
$$

where $f_{\sigma}$ is the scaling factor relative to the Minimum Mass Solar Nebula (MMSN) (Hayashi, 1981) and $\sigma_{\mathrm{M} 1}$ is the surface density scaled at $1 \mathrm{AU}$ given as

$$
\sigma_{\mathrm{M} 1}= \begin{cases}7.1 \mathrm{~g} \mathrm{~cm}^{-2} & (\text { if } a<2.7 \mathrm{AU}), \\ 30 \mathrm{~g} \mathrm{~cm}^{-2} & (\text { if } a>2.7 \mathrm{AU})\end{cases}
$$

Since it is computationally too intense to perform simulations of the entire disk, we choose annuli of planetesimals at three different regions: $a=0.1,1$, and $30 \mathrm{AU}$. The width of each annulus is set to be $0.2 a$, so the effect of radial diffusion is unimportant as long as $e<0.1$, where $e$ is the orbital eccentricity. We adopt $f_{\sigma}=1$ for the suites of simulations at 0.1 and $1 \mathrm{AU}$ while a large surface density, $f_{\sigma}=10$, is adopted for the simulations at $30 \mathrm{AU}$ to reduce accretion time. The various parameters for each suite of simulations are summarized in Table 1.

The internal density of any bodies is assumed to be $2 \mathrm{~g} \mathrm{~cm}^{-3}$. We adopt three initial masses of planetesimals: $m_{0}=10^{13}, 10^{17}$, and $10^{21} \mathrm{~g}(s=0.1,2.3$, and $49 \mathrm{~km})$ for all three regions. For these simulations, we use our hybrid code. The initial number of tracers for hybrid simulations is $N_{\text {tr }}=10000$. Simulations with larger $N_{\text {tr }}$ are computationally too intense since many embryos form near the onset of oligarchic growth. To check the resolution effect we also perform the same simulations staring with $N_{\mathrm{tr}}=1000$ for some cases. In addition to the hybrid simulations, we perform pure $N$-body simulations with $N=2000$. This number gives $m_{0}=3.1 \times 10^{23} \mathrm{~g}$ and $10^{24}$ $\mathrm{g}(s=335 \mathrm{~km}$ and $492 \mathrm{~km})$ for $a=0.1$ and $1 \mathrm{AU}$, respectively. We do not perform pure $N$-body simulations at $30 \mathrm{AU}$ since practically no collision occurs within the age of the solar system for such a small $N$.

The initial velocity dispersion $u$ is set to be the escape velocity of an individual planetesimal and the initial ratio of the rms eccentricity $\langle e\rangle^{1 / 2}$ to the rms inclination $\langle i\rangle^{1 / 2}$ is 2 . Similar results are obtained even with a lower initial $u$ because $u$ quickly increases due to viscous stirring. We do not consider any effects of a gaseous disk, such as gas drag, for comparison with our analytic 
theory. Simulations are performed at least up to the midst of oligarchic growth and each run takes typically a few cpu weeks. Some of simulations are performed twice for the same input parameters but using different random numbers for creation of the initial positions and velocities. Since the statistical variations in mass and velocity evolutions are found to be small enough, we will show results of only one simulation for each set of input parameters.

For the outcome of collisions, we adopt the merging criterion proposed by Genda et al. (2012). They showed that two colliding bodies merge if the impact velocity $v_{\text {imp }}$ is lower than the critical velocity $v_{\mathrm{cr}}$, while a collision with $v_{\mathrm{imp}}>v_{\mathrm{cr}}$ results in an inelastic rebound. If $v_{\text {imp }}$ is very large, collisional fragmentation occurs in reality (Benz and Asphaug, 1999; Leinhardt and Stewart, 2012), although we ignore this effect in our simulations as well as our analytic theory. If perfect accretion is applied, on the other hand, the masses of the smallest planetesimals unnaturally increase through mutual collisions even after their impact velocities become much larger than the mutual escape velocities. Such an effect is again not considered in the analytic theory.

Consider an impact between the target with mass $m_{i}$ and the impactor with mass $m_{j}\left(\leq m_{i}\right)$. Let the impact angle be $\theta_{\mathrm{c}}\left(\theta_{\mathrm{c}}=0\right.$ for a head-on collision). Based on thousands of SPH simulations, Genda et al. (2012) derived the formula of $v_{\text {cr }}$ as

$$
\frac{v_{\mathrm{cr}}}{v_{\mathrm{esc}}}=c_{1} \Gamma \Theta^{c_{5}}+c_{2} \Gamma+c_{3} \Theta^{c_{5}}+c_{4},
$$

where $v_{\text {esc }}$ is the mutual escape velocity, $\Gamma=\left(m_{i}-m_{j}\right) /\left(m_{i}+m_{j}\right)$, and $\Theta=1-\sin \theta_{\mathrm{c}}$. The coefficients are $c_{1}=2.43, c_{2}=-0.0408, c_{3}=1.86, c_{4}=1.08$, and $c_{5}=5 / 2$. While we use Eq. (31), our simulation results are not sensitive to choice of $v_{\mathrm{cr}} / v_{\mathrm{esc}}$ as long as it is somewhat larger than unity. The impact velocity vector is decomposed to the normal and tangential components $\left(v_{n}\right.$ and $v_{t}$ ) relative to the vector pointing toward the center of the target from the center of the impactor. If the impact is judged as a hit-and-run collision, the tangential component $v_{t}$ is assumed to be unchanged and the normal component of the post-impact relative velocity $v_{n}^{\prime}$ is given as

$$
v_{n}^{\prime}= \begin{cases}0 & \left(\text { for } v_{t}>v_{\mathrm{esc}}\right), \\ -\left(v_{\mathrm{esc}}^{2}-v_{t}^{2}\right)^{1 / 2} & (\text { otherwise }) .\end{cases}
$$

This velocity change is similar to but slightly different from that adopted by Kokubo and Genda (2010), who always set $v_{n}^{\prime}=0$ and adjust the post-impact $v_{t}$. We do not consider any mass exchange between the target and the impactor for a hit-and-run collision. Appendix E of Morishima (2015) describes in detail how hit-and-run collisions are handled in the hybrid code.

\subsection{Results}

The overall results well agree with the theoretical predictions described in Section 2. We briefly recap how oligarchic growth commences for different input parameters. Simulations at $a=30$ AU slightly suffer from resolution effects and one needs to see the results with some caution. We will discuss these effects in the next section.

Figure 1demonstrates how planetary accretion proceeds in our simulations. In the early stage, some large planetesimals grow rapidly and they locally exert velocity dispersion. Eventually embryos form and they grow with keeping some orbital separations, as typical for oligarchic growth. For simulations at $a=0.1$ and $1 \mathrm{AU}$, about ten oligarchic bodies form in the end while only one distinctively large embryo form for the run at $a=30$ AU. Late time planetary accretion at $a=30$ 

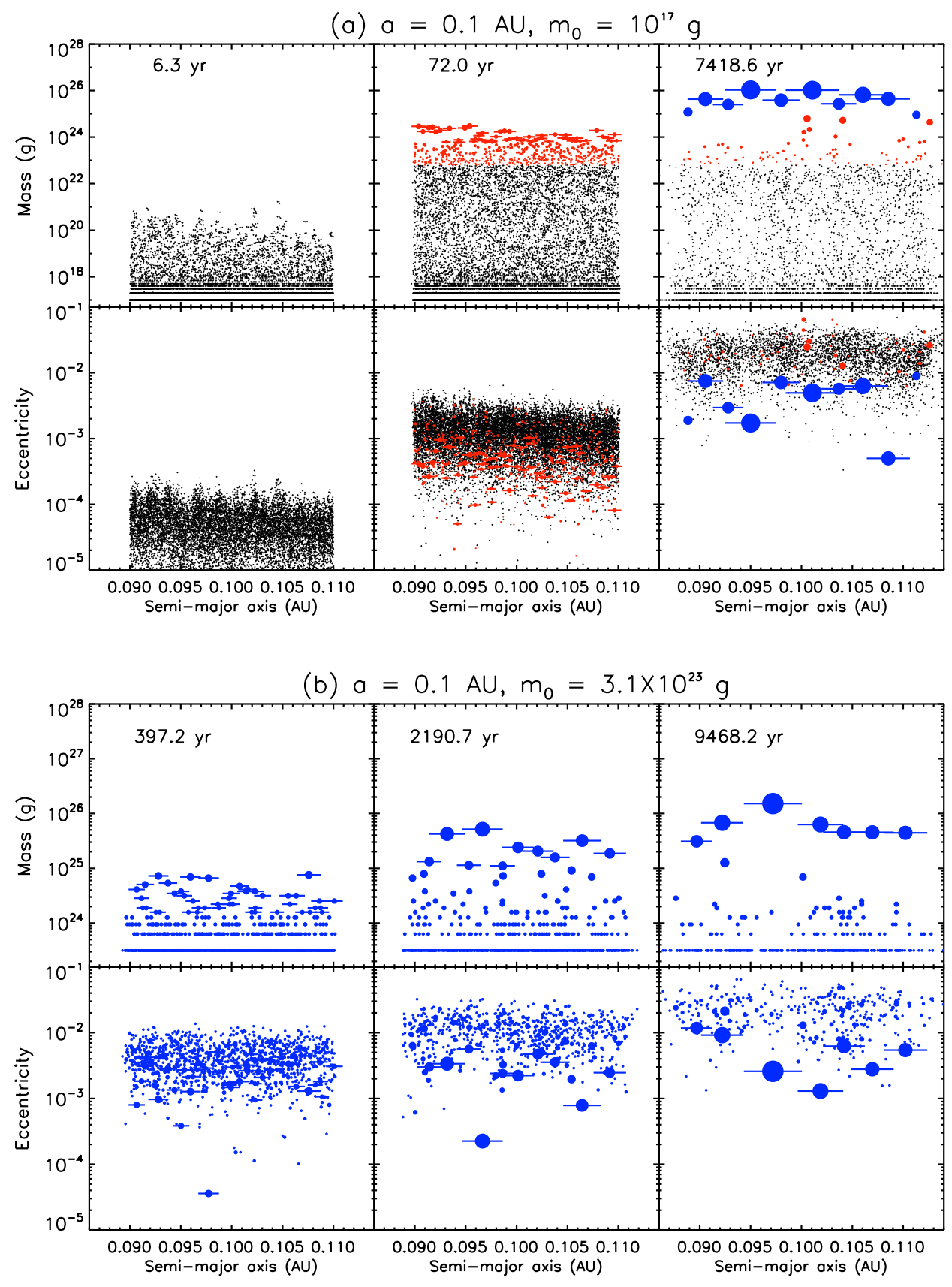

Figure 1: Snapshots of mass and eccentricity distributions for four selected simulations. For hybrid simulations $(\mathrm{a}, \mathrm{c}, \mathrm{d})$, tracers are black dots, sub-embryos $\left(m_{t 0} \leq m<100 m_{t 0}\right)$ and full embryos $\left(m \geq 100 m_{t 0}\right)$ are red and blue circles, respectively. The circle's radius for an embryo is proportional to its physical radius. For tracers, plotted masses are those of planetesimals in them, not tracers masses. The horizontal bars are given for embryos more massive than $0.2 M$ and the half length of the bar is 10 Hill radii of each embryo. 

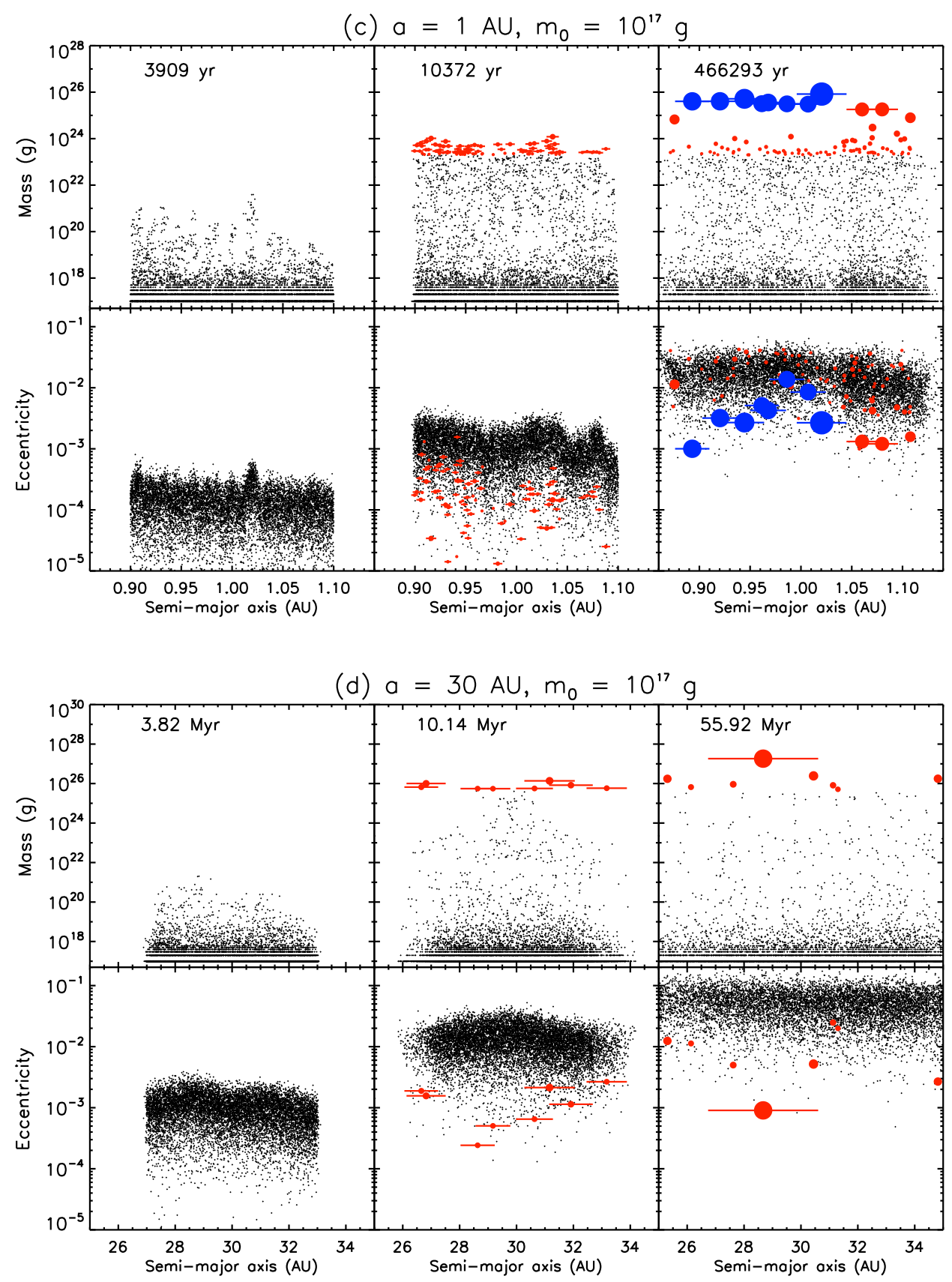

Figure 1- continue. 
(a) $a=0.1 \mathrm{AU}$

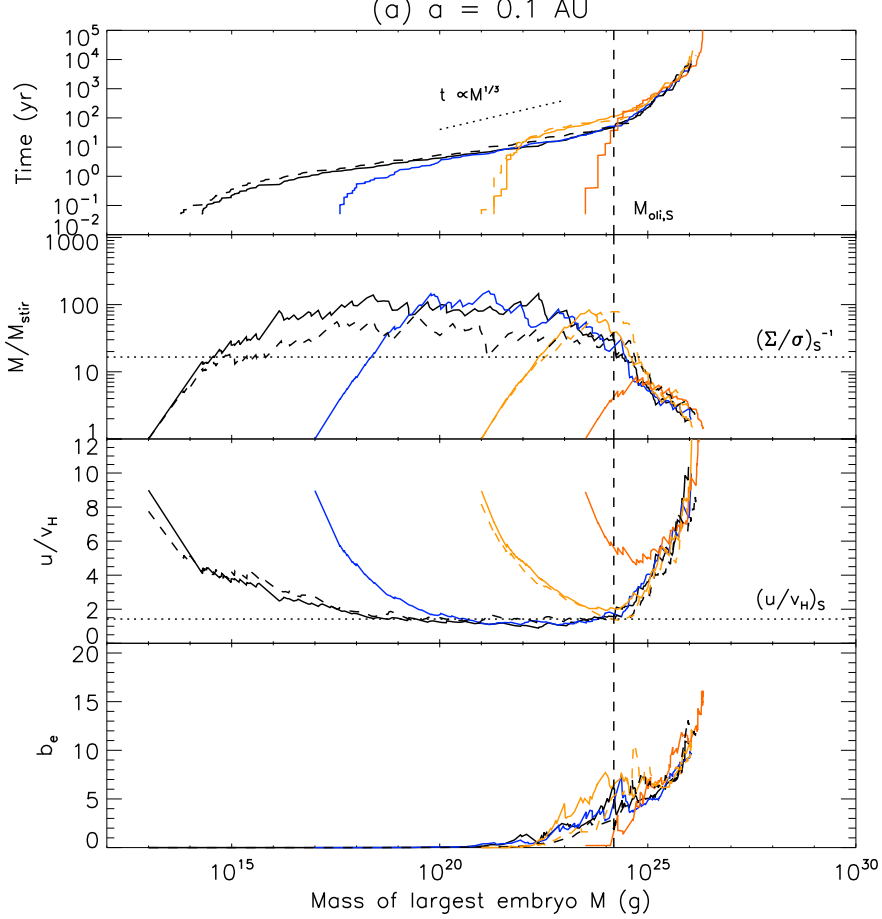

(b) $a=1 \mathrm{AU}$

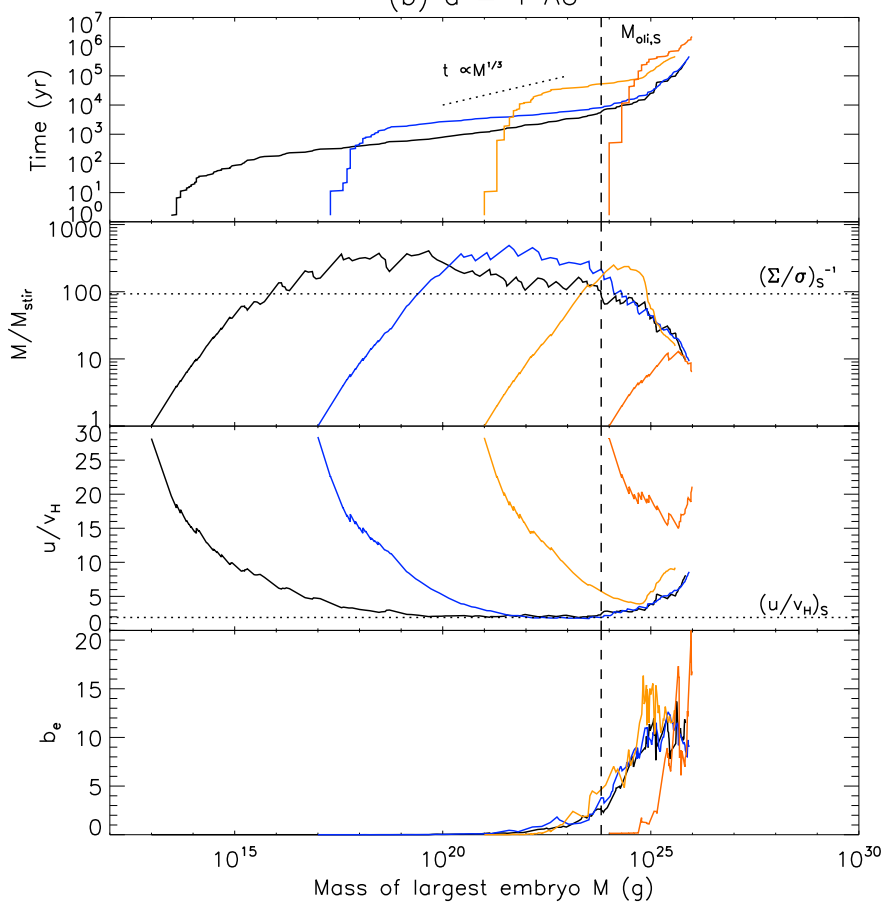

Figure 2: Time $t, M / M_{\mathrm{stir}}, u / v_{\mathrm{H}}$, and $b_{\mathrm{e}}$ as a function of largest body's mass $M$ for $a=0.1,1$, and 30 AU. For hybrid simulations $\left(m_{0}=10^{13}, 10^{17}\right.$, and $\left.10^{21} \mathrm{~g}\right), N_{\mathrm{tr}}=10000$ (solid) and 1000 (dashed). The vertical dashed line is $M_{\mathrm{oli}, \mathrm{S}}$ [Eq. [25)]. The slope for $t$ and the values for $M / M_{\mathrm{stir}}\left(=(\Sigma / \sigma)_{\mathrm{S}}^{-1}\right)$ and $u / v_{\mathrm{H}}$ expected from the theory for small $m_{0}$ are shown by the dotted lines. 


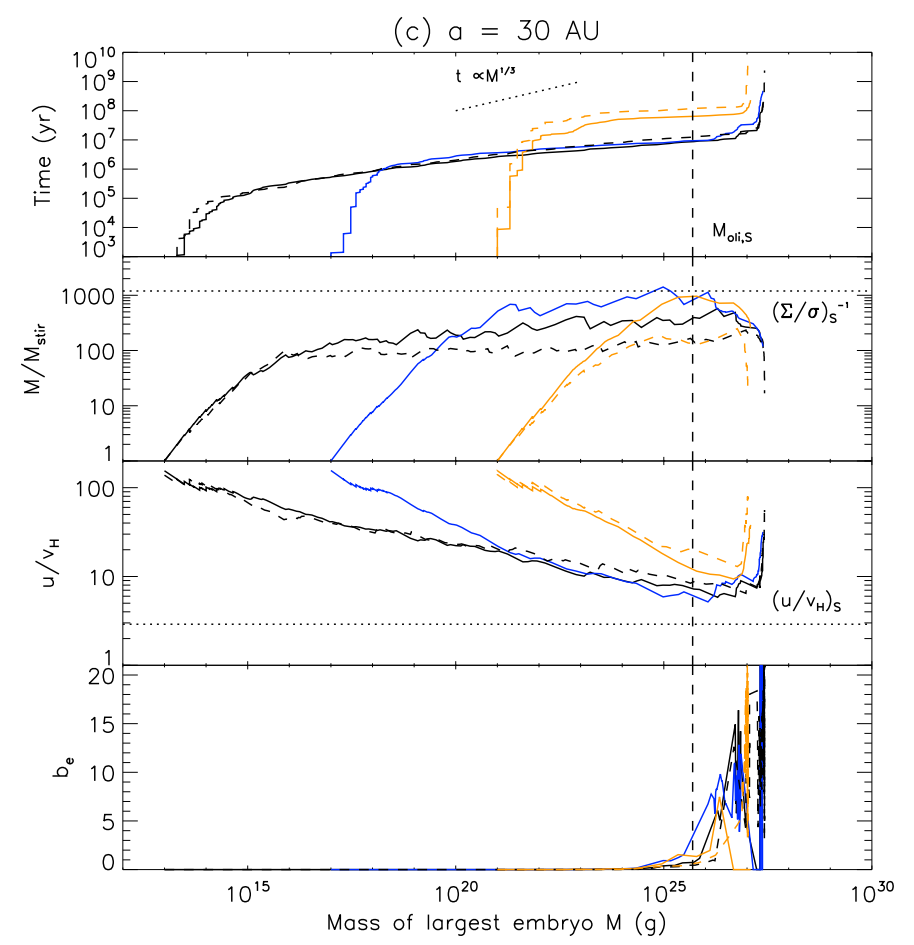

Figure 2-continue.

AU is halted primarily by depletion of the local surface density of planetesimals due to their radial diffusion, although its effect is insignificant around the onset of oligarchic growth where $M \sim M_{\text {oli }}$.

To see the time evolution of each simulation more quantitatively, we plot in Fig. 2 the time $t$, the largest bodies mass $M$ relative to the effective mass $M_{\text {stir }}\left(=\left\langle m^{2}\right\rangle /\langle m\rangle\right)$, the normalized velocity $u / v_{\mathrm{H}}$, and the normalized orbital separation of large bodies $b_{e}$ taking $M$ in the horizontal axis. We define the velocity $u / v_{\mathrm{H}}$ as

$$
\frac{u}{v_{\mathrm{H}}} \equiv \frac{\left\langle e^{2}\right\rangle_{0}^{1 / 2}+\left\langle i^{2}\right\rangle_{0}^{1 / 2}}{h_{M}}
$$

where $\left\langle e^{2}\right\rangle_{0}^{1 / 2}$ and $\left\langle i^{2}\right\rangle_{0}^{1 / 2}$ are the rms eccentricity and inclination of smallest bodies $\left(m=m_{0}\right)$ and $h_{M}$ is the reduced Hill radius of the largest body. Statistical fluctuation of $h_{M}$ is likely to be small since the mean value for the five largest bodies is found to be very close to $h_{M}$. We measure the normalized orbital separation $b_{\mathrm{e}}$ as

$$
b_{\mathrm{e}}=\frac{1}{N_{\mathrm{L}}-1} \sum_{i}^{N_{\mathrm{L}}-1} b_{i j}, \quad b_{i j}=\frac{a_{j}-a_{i}}{\left(k_{i}+k_{j}\right)\left(a_{i}+a_{j}\right) h_{i j}^{\prime} / 4},
$$

where $N_{\mathrm{L}}$ is the number of particles (embryos or tracers) with $m>0.2 M$, the index $i$ is in ascending order of $a, j=i+1$, and $k_{i}$ is the number of bodies in a tracer and unity for an embryo. We approximately applied the following form of the reduced mutual Hill radius $h_{i j}^{\prime}$ only for measuring 
$b_{\mathrm{e}}$

$$
h_{i j}^{\prime}=\left(\frac{m_{i j}}{3 M_{\odot}}\right)^{1 / 3}, \quad m_{i j}^{\prime}=2 \frac{k_{i} m_{i}+k_{j} m_{j}}{k_{i}+k_{j}} .
$$

This form is reduced to the standard reduced mutual Hill radius if both neighboring bodies are embryos $\left(k_{i}=k_{j}=1\right)$. In Eq. (34), bodies less massive than $0.2 M$ are not counted, since they are scattered by large bodies and behave like small planetesimals. Neighboring pairs with $b_{i j}>30$ are not counted since they are likely to grow in spatially different regions with little gravitational interactions.

The very beginning of planetary accretion takes place by collisions between small planetesimals which have comparable collisional and geometric cross sections. Once $M$ becomes more massive than $\sim 10 m_{0}$, runaway growth starts to occur due to gravitational focusing. With increasing $M$, $M / M_{\text {stir }}$ increases and $u / v_{\mathrm{H}}$ decreases. This accelerates the growth of the largest body.

If $m_{0}$ is small, $u / v_{\mathrm{H}}$ eventually reaches as low as unity and $M / M_{\text {stir }}$ takes its highest value which is comparable to the theoretically expected value. This low $u / v_{\mathrm{H}}$ state, or the trans-Hill stage, continues until $M$ reaches $M_{\mathrm{oli}, \mathrm{S}}$. The mass increases as $M \propto t^{3}(d R / d t=$ const $)$ as long as $u / v_{\mathrm{H}}$ is nearly fixed during this stage. Some cases have steeper dependence since $u / v_{\mathrm{H}}$ gradually decreases with $M$. As the largest body grows more massive than $M_{\mathrm{oli}, \mathrm{S}}, u / v_{\mathrm{H}}$ starts to increase. This is because now the accretion timescale of the largest body is longer than the stirring timescale. The orbital separation of large bodies normalized by their mutual Hill radius at this time is about 3-10.

If $m_{0}$ is not small, $u / v_{\mathrm{H}}$ starts to increase without decrease of $u / v_{\mathrm{H}}$ down to near unity. In this case, the planetary mass at this turnaround point is about $M_{\mathrm{oli}, \mathrm{L}}$. Similar trends are seen regardless of $a$.

In the same manner of Ormel et al. (2010b), we regard $M$ at the minimum $u / v_{\mathrm{H}}$ as the planetary mass $M_{\text {oli }}$ at the onset of oligarchic growth. This mass for each simulation is shown as a triangle in Fig. 3, and the minimum $u / v_{\mathrm{H}}$ is plotted in Fig. 4. If $m_{0}$ is small, this mass may not be very meaningful since $u / v_{\mathrm{H}}$ fluctuates around a similar value for a wide range of $M$. Hence we also measure the low velocity range in the $M$-space in which $u / v_{\mathrm{H}}<\operatorname{MIN}\left(u / v_{\mathrm{H}}\right)+\Delta$. The offset $\Delta$ should be as small as possible but large enough compared with the fluctuation amplitude in $u / v_{\mathrm{H}}$. We choose $\Delta=\left(u / v_{\mathrm{H}}\right)_{\mathrm{S}}=\alpha^{-1 / 8}$, and the range is shown by the error bars for each simulation in Fig. 3. This range can be roughly regarded as the trans-Hill stage, although the width of the range in the $M$-space is overestimated due to a finite $\Delta$. We find that $M_{\text {oli }}$ and the minimum $u / v_{\mathrm{H}}$ from the numerical simulation reasonably agree with the theoretical prediction. Even if the agreement of $M_{\text {oli }}$ is not very good for some simulations with low $m_{0}$, the range of $M$ in the low velocity stage well encompasses the theoretically predicted $M_{\text {oli }}$. For low $m_{0}$, the trans-Hill stage begins when $M$ reaches $\sim M_{\text {tran }}[$ Eq. (23)] $]$ as predicted, except for the cases of $a=30 \mathrm{AU}$.

Figure 5 shows snapshots of the mass and velocity distributions for selected simulations. During runaway growth, $q \simeq-2.7$ to -2.5 , as reported in the literature (Kokubo and Ida, 1996; Barnes et al., 2009; Ormel et al., 2010b). During the trans-Hill stage that appears in simulations with small $m_{0}, q$ for the massive bodies is about -2.0 (a flat line in the top panel). The mass fraction contained in the most massive bodies, or the efficiency $\Sigma / \sigma$ at the onset of oligarchic growth well agrees with the theoretical prediction. The dependence of $\Sigma / \sigma$ on $\alpha$ for small $m_{0}$ is weaker than $\propto \alpha$ predicted by Eq. (12) but close to $\propto \alpha^{3 / 4}$ given by Eq. (24). During the oligarchic growth stage, oligarchic bodies efficiently merge together while the mass distribution slope of planetesimals $\left(m<M_{\text {oli }}\right)$ retains. 


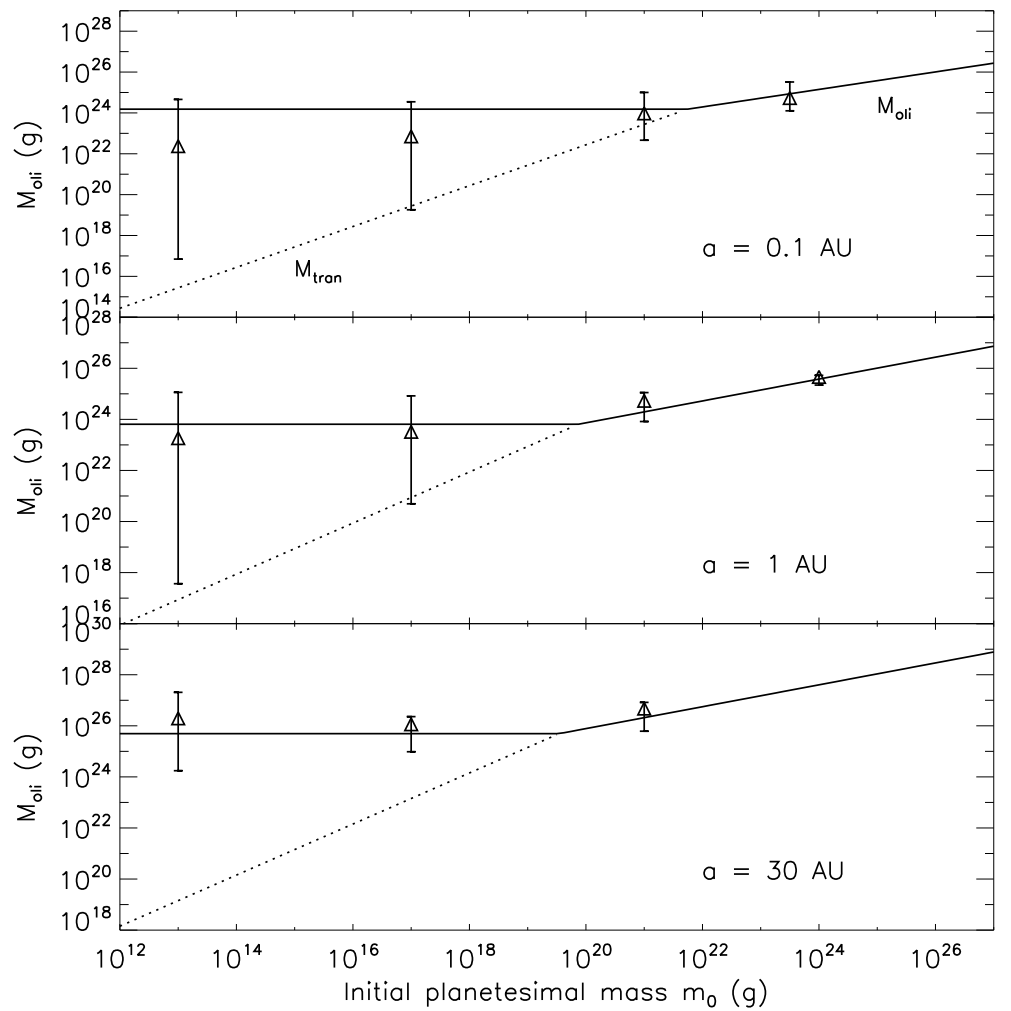

Figure 3: Planetary mass $M_{\mathrm{oli}}$ at the onset of oligarchic growth as a function of initial planetesimal mass $m_{0}$ for $a=0.1,1$, and $30 \mathrm{AU}$. The values from numerical simulations are shown by triangles with error bars (see the text how we derived them) while the theoretical prediction [Eq. [26] ] is shown by a solid line in each panel. The dotted line is $M_{\text {tran }}[$ Eq. [23)] for $q=-2.5$. 


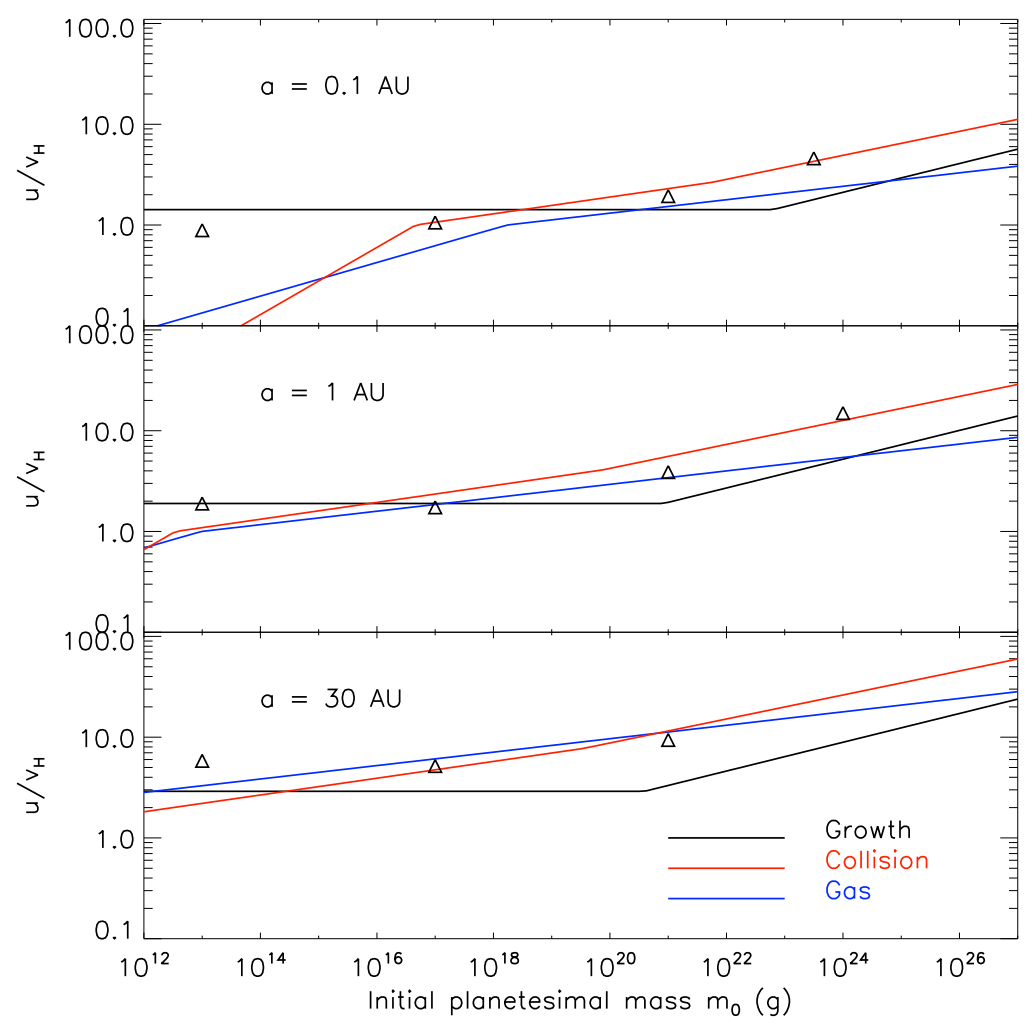

Figure 4: Velocity dispersion at the onset of oligarchic growth or minimum $u / u_{\mathrm{H}}$. Triangles are the values from simulations while black curves are theoretical predictions [Eq. [27] ] in the case without any damping force. The equilibrium velocities determined by balance between viscous stirring and collisional damping are shown by red curves. The equilibrium velocities determined by balance between viscous stirring and gas drag are shown by blue curves. See Section 4.5 for the red and blue curves. 

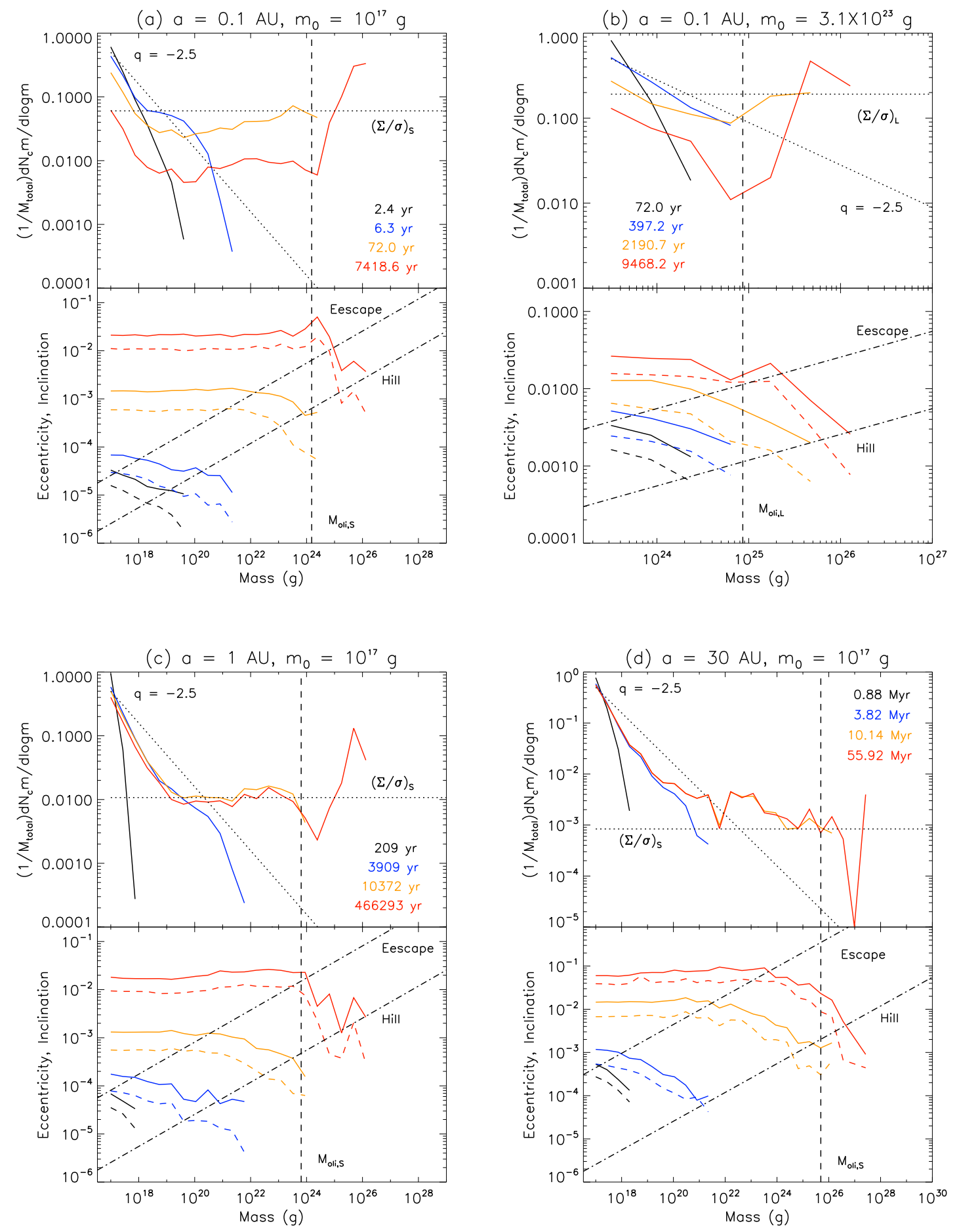

Figure 5: Snapshots of the mass and velocity distributions for four simulations selected in Fig. 1 The vertical dashed line is the predicted $M_{\mathrm{oli}}$. In the top panel, the flat dotted lines is the predicted $\Sigma / \sigma$ at the onset of oligarchic growth and the inclined dotted line shows a slope of $q=-2.5$. In the bottom panel, the colored curves are the eccentricity (solid) and inclination (dashed) distributions and the two dot-dash lines correspond to the escape and hill velocities at that mass. 

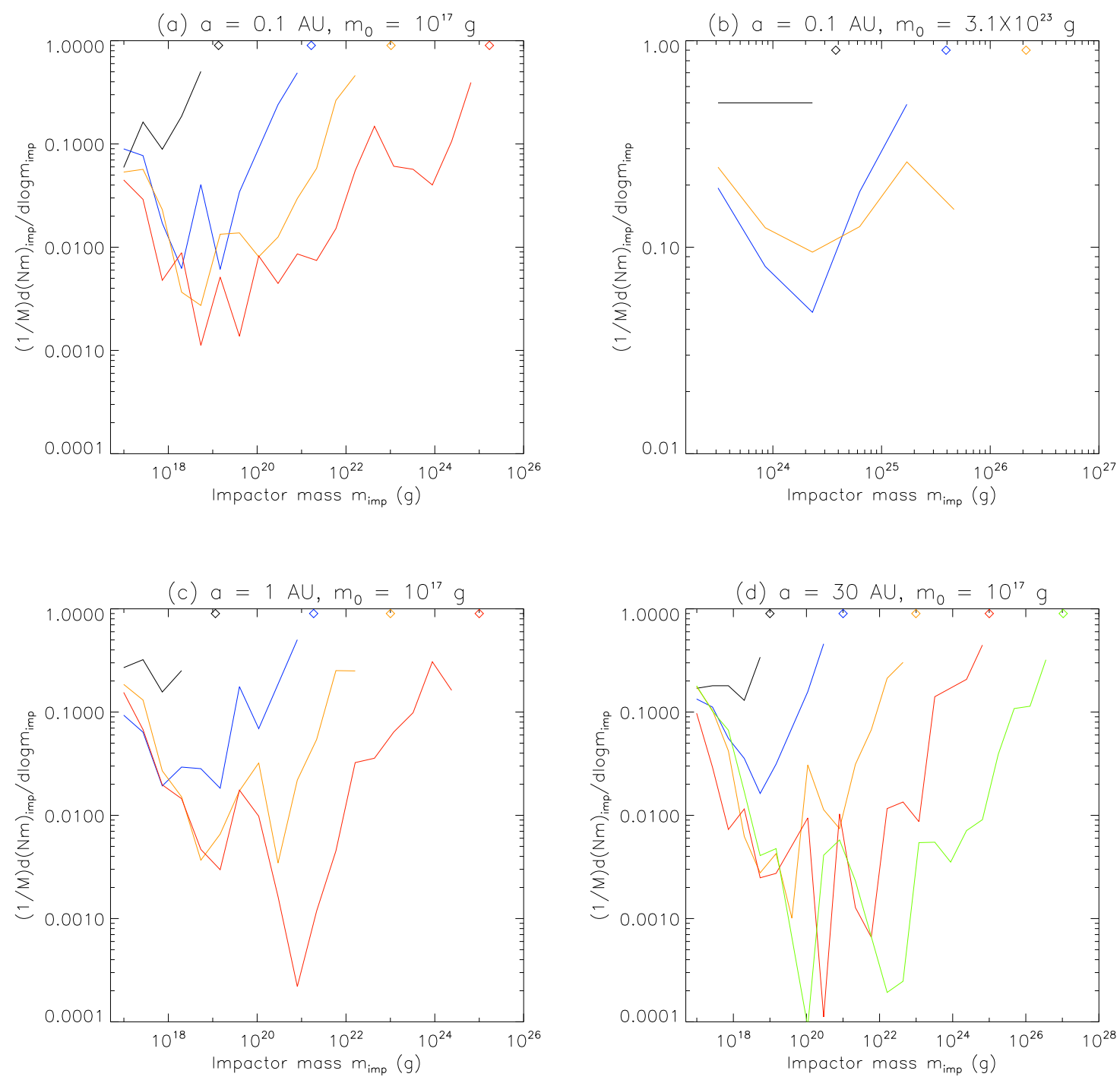

Figure 6: Mass distribution of impactors that collided with the largest body until a certain time for the same simulations selected in Fig. 1 The largest body is selected by its mass at the end of each simulation. The largest body's mass at each time is shown by a diamond and the corresponding impactor's distribution is shown by a solid curve with the same color of the diamond. 
Due to the balance between viscous stirring and dynamical friction, the rms eccentricity and inclination distributions follow $e, i \propto m^{-1 / 4}$ during the runaway and trans-Hill stages for bodies with their escape velocities larger than the velocity dispersion (see the last paragraph of Section 2.2). The velocity is nearly flat for smaller bodies. In the very early stage of runway growth $(q<-3)$, the energy equipartitioning is nearly achieved $\left(e, i \propto m^{-1 / 2}\right)$ since the heating effect of dynamical friction (ignored in Section 2.2) is more important than viscous stirring of large bodies. For the same reason, the energy equipartitioning is generally achieved for oligarchic bodies, as their mutual stirring is inefficient.

Figure 6 shows the mass distribution of impactors that merge with the largest body. We find that the contribution of merging with similar-sized bodies is comparable to or somewhat larger than the contribution of small bodies in all the simulations at any time. During the trans-Hill stage, efficient merging between large bodies is realized as they have very low inclinations due to inefficient vertical stirring (see Fig. 5). A similar result is found in the simulation of Ormel et al. (2010a).

\subsection{Resolution effects of hybrid simulations}

For the simulations at $a=0.1 \mathrm{AU}, M_{\text {oli }}$ derived from numerical simulations is almost in convergence, as we see good agreements in $u / v_{\mathrm{H}}$ between simulations with $N_{\mathrm{tr}}=1000$ and 10000 (Fig. 2a). This is expected since $m_{t 0}<M_{\text {oli,S }}$ for both simulations. We also performed simulations with $N_{\mathrm{tr}}=10^{5}$ for some cases only up to midst of the trans-Hill stage. We found that $u / v_{\mathrm{H}}$ at its minimum is slightly lower than that for $N_{\text {tr }}=10000$ and this may cast doubt on numerical convergence. However, the mass spectrum falls off very steeply $(q<-3)$ in the most massive tail for $N_{\mathrm{tr}}=10^{5}$ so the largest bodies do not significantly contribute to stirring. If we interpret $h_{M}$ as the effective reduced Hill velocity of massive bodies that contribute to stirring the most for consistency with the theory, $u / v_{\mathrm{H}}$ at its minimum is already in good convergence for $a=0.1 \mathrm{AU}$.

The simulations at $a=30 \mathrm{AU}$, on the other hand, do not reach convergence, as $M_{\text {oli }}$ and $u / v_{\mathrm{H}}$ at its minimum significantly decrease with increasing $N_{\mathrm{tr}}$ (Fig. 2 $\mathrm{k}$ ). This is also expected since $m_{t 0}>M_{\mathrm{oli}, \mathrm{S}}$ for $N_{\mathrm{tr}}<10000$. However, we expect that the simulation with $N_{\mathrm{tr}}=10000$ is near convergence, as it gives $M_{\text {oli }}$ only slightly larger than the prediction.

This argument seems to be supported by other studies. The coagulation simulation of Ormel et al. (2010a) at $a=35$ AU with $s=1 \mathrm{~km}\left(m_{0} \simeq 10^{16} \mathrm{~g}\right)$ (their Fig. 16) shows that $u / v_{\mathrm{H}}$ reaches its minimum value, $\simeq 3$, at $M \sim 10^{23} \mathrm{~g}$ and the trans-Hill stage continues until $u / v_{\mathrm{H}}$ starts to increase around $M \sim 10^{24} \mathrm{~g}$. This mass is quite close to $M_{\mathrm{oli}, \mathrm{S}}$ that we predict for $\sigma$ and $m_{0}\left(<m_{0, \text { crit }}\right)$ that they employed. The minimum value of $u / v_{\mathrm{H}}$ is about 5 in our simulations for $m_{0}<m_{0 \text {,crit }}$ (Fig. 2r) and is close to their value. The simulation of Shannon et al. (2015) at $a=45 \mathrm{AU}$ with $s=1 \mathrm{~km}$ also shows a result similar to Ormel et al. (2010a). The efficiency $\Sigma / \sigma$ of $\sim 10^{-3}$ found by Schlichting and Sari (2011) and Shannon et al. (2015) is also close to ours (Fig. $5 \mathrm{~d}$ d).

\section{Discussion}

\subsection{Planetary accretion overview}

Figure 7 shows the planetary mass at the onset of oligarchic growth as a function of semimajor axis for the surface densities of 1 and 10 times of the MMSN. The overall growth of planets is described as follows. Runaway growth first commences after the mass of the largest body $M$ becomes more massive than about 10 times the initial mass of planetesimals $m_{0}$. During runaway 


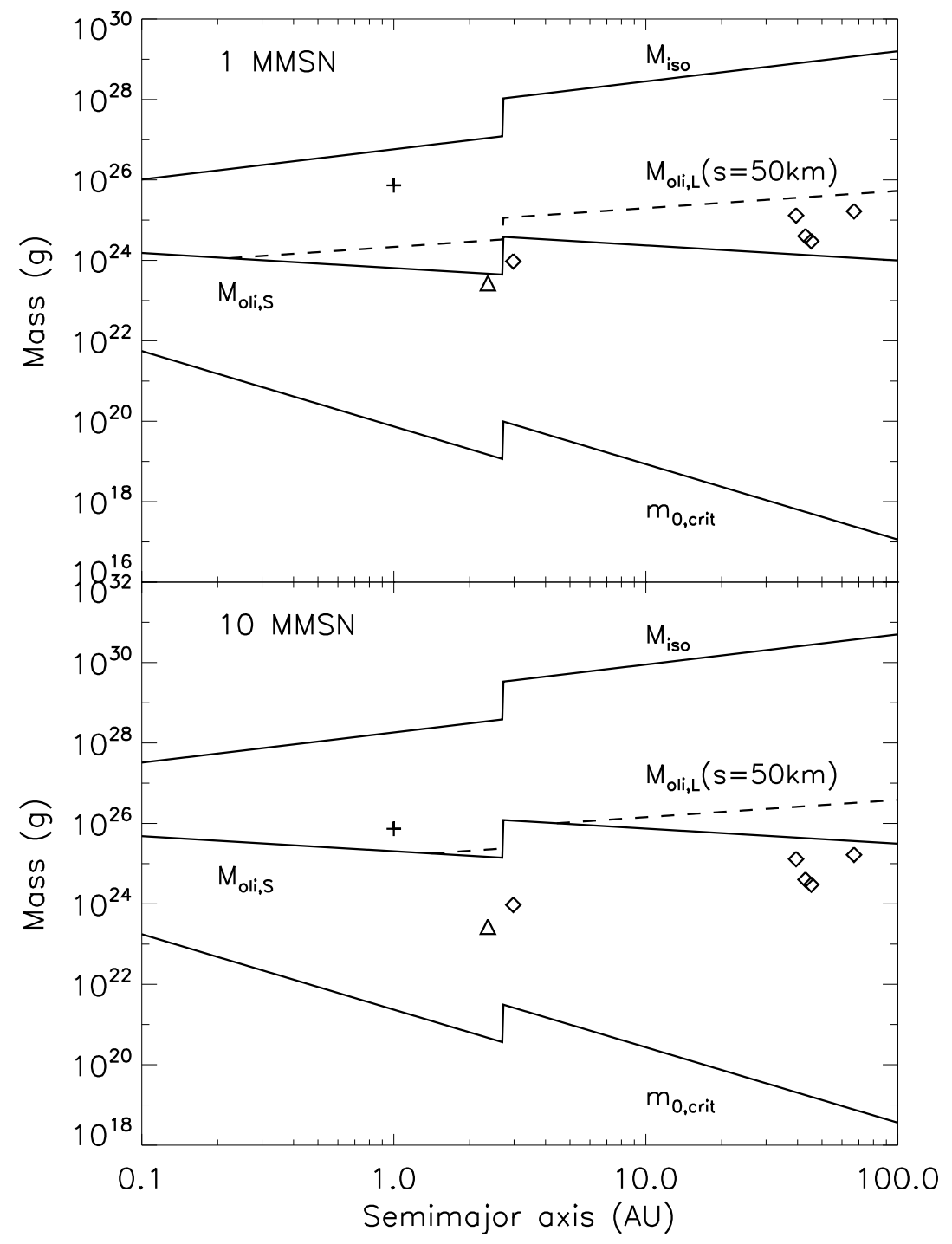

Figure 7: Planetary masses at the onset of oligarchic growth $M_{\mathrm{oli}, \mathrm{S}}$ for $m_{0}<m_{0, \text { crit }}$ and $M_{\mathrm{oli}, \mathrm{L}}$ for $s=50 \mathrm{~km}$ and $q=-2.5$. The isolation mass $M_{\text {iso }}$ and the critical planetesimal mass $m_{0, \text { crit }}$ are also shown. The upper and lower panels show the cases of $f_{\sigma}=1$ and 10, respectively. The diamonds indicate the dwarf planets (Ceres, Pluto, Haumea, Makemake, and Eris in ascending order of $a$ ). The plus and the triangle indicate Moon and Vesta. 
growth, $u / v_{H}$ decreases with increasing $M$. If $m_{0}<m_{0, \text { crit }}$ [Eq. (28)], the trans-Hill stage appears when $u / v_{H}$ reaches near unity $\left(\alpha^{-1 / 8}\right)$. The mass at the onset of the trans-Hill stage is given by $M_{\text {tran }}$ [Eq. (23)]. The trans-Hill stage stage ends and oligarchic growth commences when $M$ reaches to $M_{\mathrm{oli}, \mathrm{S}}$ [Eq. (25)], which is independent of $m_{0}$. If $m_{0}>m_{0, \text { crit }}$, on the other hand, oligarchic growth commences directly after runaway growth. In this case, the planetary mass at the onset of oligarchic growth is given by $M_{\text {oli, } \mathrm{L}}$ [Eq. (14)], which is proportional to $m_{0}^{3 / 7}$ for $q=-2.5$. When $M=M_{\mathrm{oli}, \mathrm{L}}, u / v_{H}$ takes its minimum $\left(>\alpha^{-1 / 8}\right)$ given by Eq. (16). In Fig. 7 , the dashed line shows $M_{\text {oli,L }}$ for $s=50 \mathrm{~km}$.

Regardless of $m_{0}, u / v_{H}$ increases with $M$ during oligarchic growth. Oligarchic growth ends when planets sweep up all surrounding planetesimals and the planetary mass at that time is the isolation mass given by $M_{\text {iso }}$ [Eq. (9)]. Before planetary accretion completes, largest remnant planetesimals which are less massive than $\sim M_{\text {oli }}$ have a power-law exponent of $q \simeq-2.0$ for $m_{0}<m_{0, \text { crit }}$ and $q \simeq-2.7$ to -2.5 for $m_{0}>m_{0, \text { crit }}$.

\subsection{Dwarf planets in asteroid and Kuiper belts}

In Fig. 7, we overplot the masses of Vesta (Russell et al., 2012) and the five dwarf planets recognized by the IAU as of January 2016: Ceres (Thomas et al., 2005, and references therein), Pluto (Stern et al., 2015), Haumea (Ragozzine and Brown, 2009), Makemake (Brown, 2013, we assumed $\rho=2.3 \mathrm{~g} \mathrm{~cm}^{-3}$ ), and Eris (Brown and Schaller, 2007). Their masses are found to be comparable to $M_{\text {oli }}$ for the solid surface density of the MMSN in a reasonable range of $s$. This implies that they are likely to be the largest remnant planetesimals that failed to become planets while large oligarchic bodies $\left(m>M_{\text {oli }}\right)$ were efficiently removed through collisional merging with planets during oligarchic growth. This argument is probably applied to asteroids. For KBOs, their accretion might be simply incomplete due to a long accretion timescale, although Pluto and Eris might have entered oligarchic growth as indicated from their separations from a continuous size distribution (Fraser et al., 2014; ; Adams et al., 2014).

In the following, we will have additional discussions for their accretion histories constrained from the mass distribution slopes. The power-law exponent $q$ for the mass distribution that we use is given by $q=5 \alpha_{\mathrm{H}} / 3+1$, where $\alpha_{\mathrm{H}}$ is the power-law exponent for the differential distribution of absolute luminosity function (H-distribution), provided that the surface albedo and the internal density are independent of size. In the following, we convert the observational $\alpha_{\mathrm{H}}$ to $q$ using this relationship. We do not discuss the mass distribution of objects smaller than $\sim 50 \mathrm{~km}$ in radius as they are likely to be collisional fragments (Bottke et al., 2005; Campo Bagatin and Benavidez, 2012).

\subsubsection{Asteroid belt}

Main asteroids have a power-low exponent of $q \simeq-2.0$ for $m>10^{21} \mathrm{~g}(s \simeq 50 \mathrm{~km})$ while a shallower slope is seen for less massive asteroids (Jedicke et al., 2002). The size at the bump is generally considered to be the initial size of planetesimals (Morbidelli et al., 2009). If planetary accretion starts from equal-mass planetesimals with $s \sim 50 \mathrm{~km}$, however, large remnant planetesimals $\left(m<M_{\text {oli, } \mathrm{L}}\right)$ turn out to have a mass distribution slope of $q \simeq-2.5$, which is steeper than the observed slope. This occurs, since $m_{0}>m_{0, \text { crit. }}$. If small initial planetesimals with $m_{0}$ lower than $m_{0 \text {,crit }}$ are employed, the shallow slope $(q \simeq-2.0)$ can be reproduced, but not the bump at $10^{21} \mathrm{~g}$. To solve this problem, Morbidelli et al. (2009) proposed that the size distribution profile of large asteroids up to Ceres was directly inherited from planetesimal formation. 


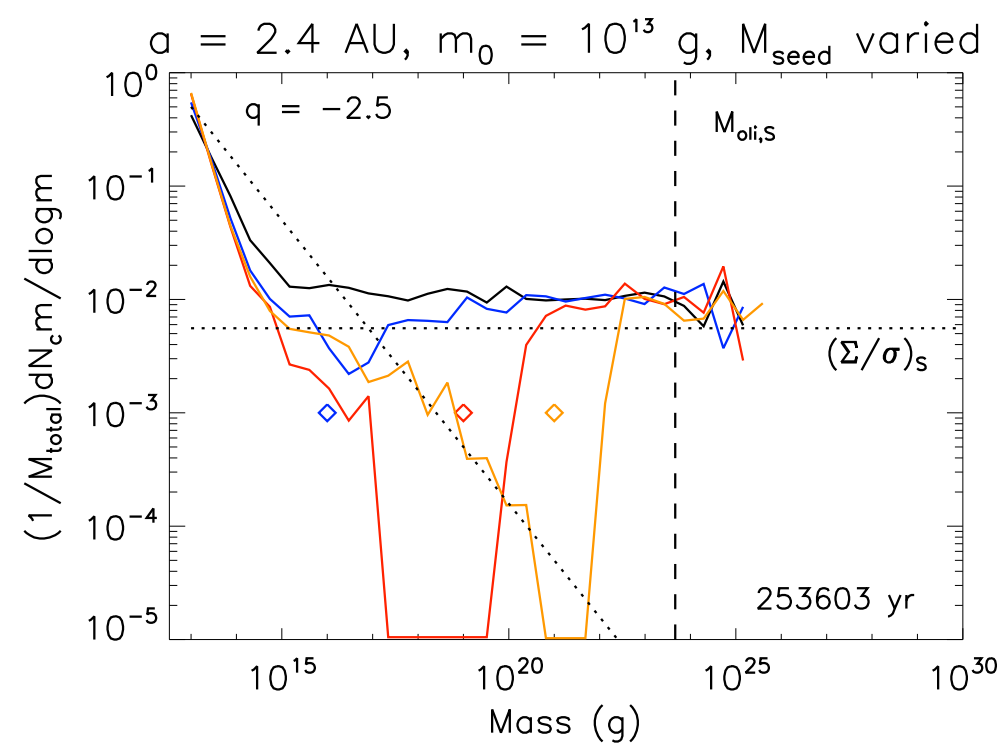

Figure 8: Mass distribution for simulations at $a=2.4 \mathrm{AU}$ and $m_{0}=10^{13} \mathrm{~g}$ with asteroid seeds. The initial mass fraction of asteroid seeds is $0.1 \%$ (shown as diamonds) and the mass of each seed is $10^{16} \mathrm{~g}$ (blue), $10^{19} \mathrm{~g}$ (red), and $10^{21} \mathrm{~g}$ (orange). The black solid curve shows the case without seeds. The initial inner and outer edges of the planetesimal disk are 2.16 and $2.64 \mathrm{AU}$. We adopt $f_{\sigma}=1, N_{\mathrm{tr}}=10000$, and $\delta t=18$ days.

Weidenschilling (2011) performed simulations similar to Morbidelli et al. (2009) while expanding a range of parameters. He found that both the bump at $10^{21} \mathrm{~g}$ and the shallow slope of large asteroids can be reproduced if the initial planetesimal size $s$ is as small as $100 \mathrm{~m}$. The bump at $10^{21} \mathrm{~g}$ was not reproduced in our simulation at $1 \mathrm{AU}$ with $m_{0}=10^{13} \mathrm{~g}(\mathrm{~s} \simeq 100 \mathrm{~m})$ even adopting $N_{\text {tr }}=10^{5}$ and similar results are seen in additional simulations at $a=2.4 \mathrm{AU}$ (Fig. 8). This may indicate that collisional fragmentation and gas drag taken into account by Weidenschilling (2011) but ignored in our simulations are important or that resolutions of our simulations are still insufficient.

Nevertheless, we find that outcome similar to simulations of Weidenschilling (2011) can be reproduced by introducing a small mass fraction of asteroid seeds while keeping small initial planetesimals. Figure 8 shows the mass distributions at the end of simulations with varying the mass of each asteroid seed $M_{\text {seed }}$. We find that the mass distribution for $M_{\text {seed }} \simeq 10^{19} \mathrm{~g}$ matches with the observation as a bump (or a shoulder in our plot) is produced at $\sim 100 M_{\text {seed }}$. Once the mass fraction of large bodies reaches the theoretical prediction $(\Sigma / \sigma)_{\mathrm{S}}$ due to their growth, the nature of their subsequent growth is very similar to that seen in the simulation starting with equal-mass planetesimals. This naturally produces the mass distribution of $q \simeq-2.0$. The important parameter to reproduce the mass distribution of asteroids in our simulations (Fig. 8) is $M_{\text {seed }}$, not $m_{0}$. Even for lower $m_{0}$, the same bump mass and the same slope for large bodies are expected. The simulations of Weidenschilling (2011) and ours imply that the fundamental point to reproduce the bump is formation of massive runaway bodies before they start to gravitationally stir small planetesimals. 


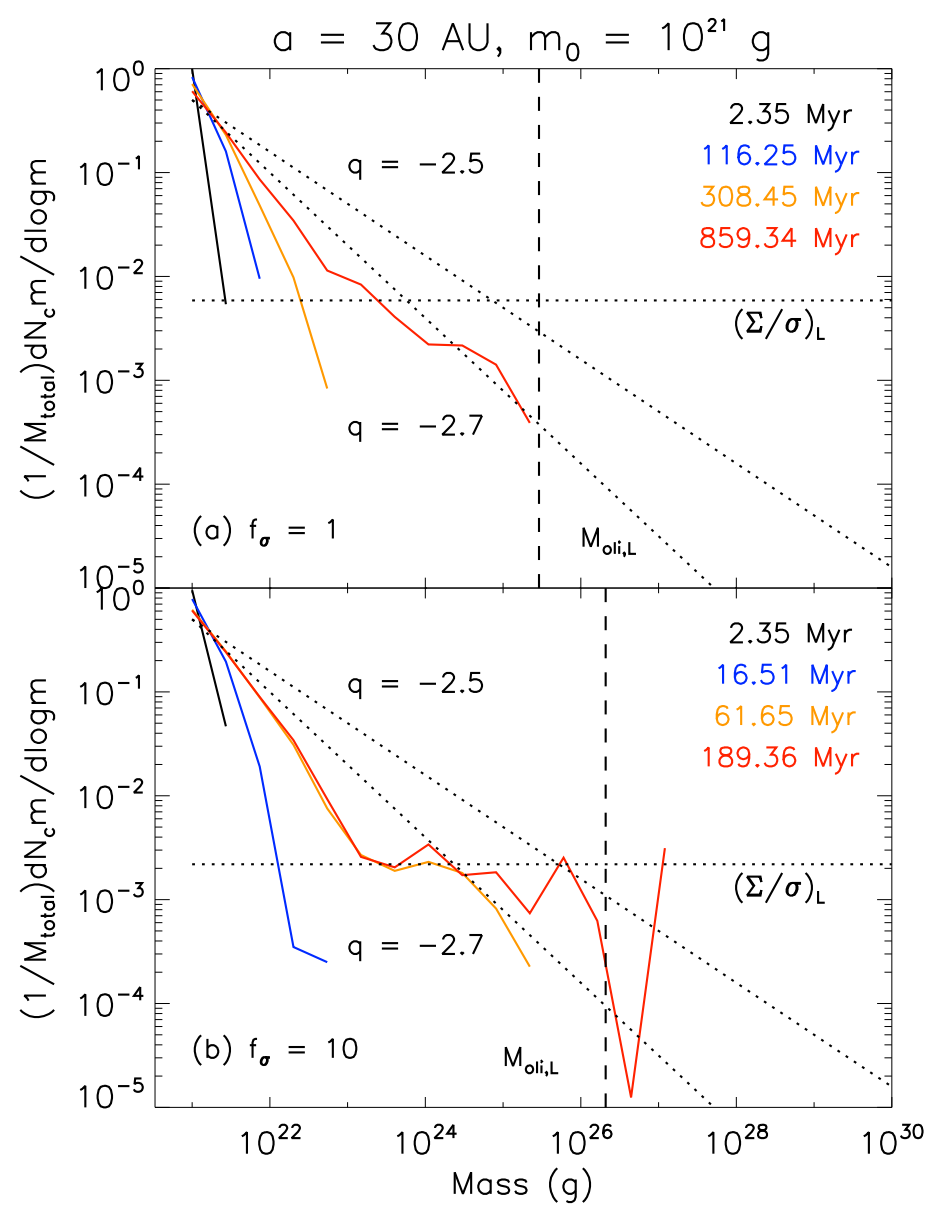

Figure 9: Mass distribution for $a=30 \mathrm{AU}$ and $m_{0}=10^{21} \mathrm{~g}$. The surface density factor $f_{\sigma}$ is (a) 1 and (b) 10 . The inclined dotted lines are slopes of $q=-2.5$ and -2.7 . 


\subsubsection{Kuiper belt}

KBOs are classified into the dynamically excited hot population with large orbital inclinations and eccentricities and the cold population with low inclinations and eccentricities (Bernstein et al., 2004). Similarly to asteroids, the both hot and cold populations of KBOs have the bump at $m \sim 10^{21} \mathrm{~g}$ in their mass distributions. The power-law exponents for bodies more massive than the bump mass are $q=-2.45_{-0.11}^{+0.32}$ for the hot population and $q=-3.5_{-0.7}^{+0.3}$ for the cold population (Fraser et al., 2014). The slopes of the absolute brightness magnitudes (Petit et al., 2011; Adams et al., 2014; Fraser et al., 2014) with corrections due to distances of KBOs are steeper than those of the apparent magnitudes (Bernstein et al., 2004; Fraser et al., 2010). Fraser et al. (2014) found that after taking into account the bimodal albedo distribution of hot KBOs, their size distribution is very similar to that for Jupiter's trojans $(q=-2.67 \pm 0.33)$. This agreement strongly supports the idea of the Nice model that trojans were captured during dynamical instability of the giant planets (Morbidelli et al., 2005). Hot KBOs are likely to have been injected to the current orbits during the instability (Malhotra, 1993; Hahn and Malhotra, 2005; Levison et al., 2008; Lykawka and Mukai, 2008; Nesvorný and Morbidelli, 2012).

The mass distribution slope and the bump mass of hot KBOs can be naturally explained if $m_{0}$ is the bump mass as $m_{0}>m_{0, \text { crit }}$. Figure 9 shows the mass distribution for the simulation with $m_{0}=10^{21} \mathrm{~g}$ at $a=30 \mathrm{AU}$. A simulation with $f_{\sigma}=1$ is performed in addition to the case with $f_{\sigma}=10$. The slope from the simulation is consistent with the observed slope $q \simeq-2.7$ after the albedo correction, particularly if $f_{\sigma}=1$. The timescale for the largest body's mass to reach the mass of Pluto $\left(1.3 \times 10^{25} \mathrm{~g}\right)$ is $60 \mathrm{Myr}$ for $f_{\sigma}=10$. If KBOs grew for $\sim 700 \mathrm{Myr}$ as suggested by the Nice model (Gomes et al., 2005), a lower surface density down to $f_{\sigma} \sim 1$ is acceptable. Our simulations are rather local while KBOs might have formed in a wide range of $a$. In this case, the mass distribution of KBOs is a superposition of those from different regions. Such a scenario was examined by Kenyon and Bromley (2012). Their results still imply that the steep slope and the bump at $10^{21} \mathrm{~g}$ are difficult to be reproduced if $m_{0}$ is lower than $m_{0 \text {,crit }}$ (see their Fig. 14).

Formation of cold KBOs by standard collisional merging is problematic because of their long growth timescale. The blue curve at $t=16.51 \mathrm{Myr}$ in Fig. 9b is similar to the slope of cold KBOs. Since the current cold population has $f_{\sigma} \sim 10^{-5}$ (Fraser et al., 2014), the timescale to produce the blue curve is $\sim 10^{13}$ yr or $3-4$ orders of magnitude longer than the age of the solar system even at $30 \mathrm{AU}$ (while cold KBOs locate at $a \sim 45 \mathrm{AU}$ ). The size distribution of cold KBOs seems primordial reflecting their formation from dust. They probably formed in situ and experienced few subsequent collisions (Batygin et al., 2011; Parker and Kavelaars, 2012).

\subsection{Planetesimal-driven migration}

Minton and Levison (2014) showed five conditions for planetesimal-driven migration (PDM) to be triggered. Using our symbols, these conditions are expressed as (1) $\sigma / \Sigma>3,(2) M / M_{\text {stir }}>100$, (3) $u / v_{\mathrm{H}}<5$, (4) $M \geq 5 M_{\text {near }}$ and (5) $t_{\text {mig }}<(d \log R / d t)^{-1}$. Here $M_{\text {near }}$ is the largest mass of nearby protoplanets in the scattering zone of the protoplanet of interest and $t_{\mathrm{mig}}$ is the migration timescale. The condition (4) is probably replaced by $M>M_{\text {oli }}$ as massive nearby protoplanets inevitably exist $\left(b_{\mathrm{e}}<3\right)$ before the onset of oligarchic growth. The condition (5) represents a constraint that the protoplanet does not encounter with other massive protoplanets during PDM. The conditions (1) and (5) are fulfilled at large $a$. The present study shows that the conditions (2), (3), and (4) are simultaneously fulfilled when $M \sim M_{\text {oli }}$. Minton and Levison (2014) examined when all the conditions are fulfilled by performing simulations of planetary accretion around 1 AU. The masses of migration candidates were found to be 0.1 to 10 times the lunar mass for 


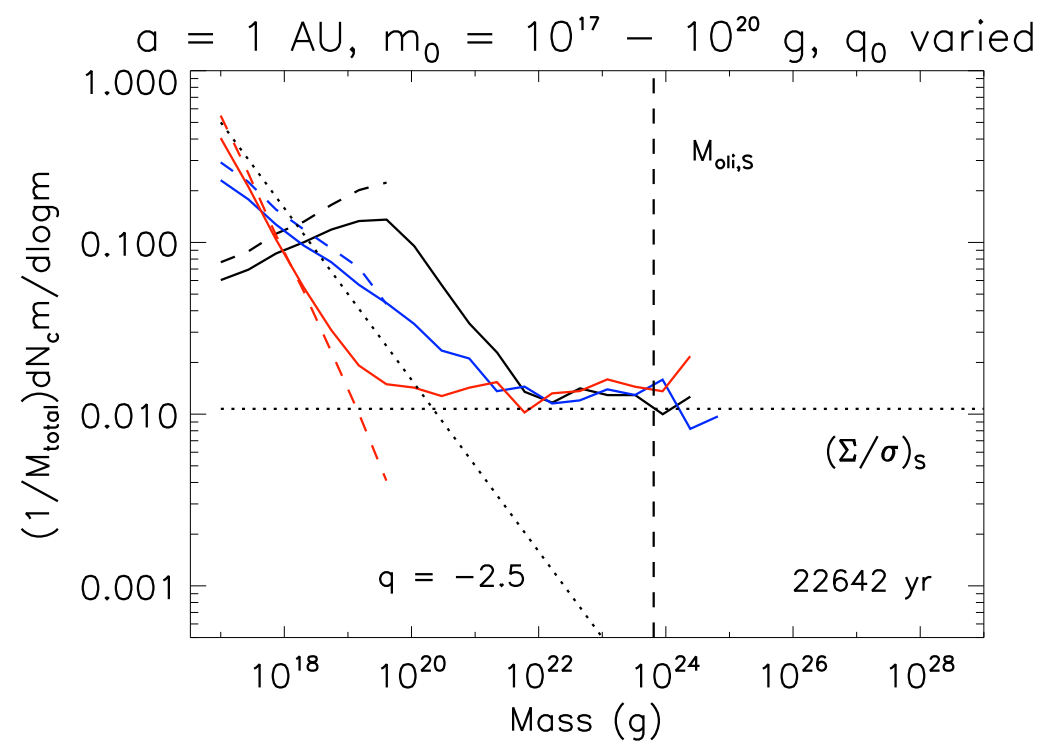

Figure 10: Effects of initial mass distribution for $a=1 \mathrm{AU}$ and $f_{\sigma}=1$. Initial planetesimal masses range between $10^{17}$ and $10^{20} \mathrm{~g}$ and the initial power-law index $q_{0}$ is -1.8 (black), -2.3 (blue), and -2.8 (red). For each case, the initial and final mass distributions (at $t=0$ and $22642 \mathrm{yr}$ ) are shown by dashed and solid curves. The initial velocity dispersion is the escape velocity of the smallest body.

$f_{\sigma}=1.1-4.5$ and $s=25-50 \mathrm{~km}$. This lowest mass of migration candidates is close to $M_{\text {oli }}$ estimated by the present study (Fig. (7). Therefore, PDM is likely to occur during early to middle oligarchic growth before the largest protoplanet is caught up by other nearby protoplanets in mass and at large $a(>1 \mathrm{AU})$. Our study also showed that the condition (3) can be better fulfilled for smaller $m_{0}$, consistent with the finding of Minton and Levison (2014).

PDM is not important in our simulations at 0.1 and $1 \mathrm{AU}$ (Fig. 1). On the other hand, we see embryos migrate back and force between the inner and outer edges of the planetesimal annulus and migration enhances mutual merging between embryos for the simulations at $a=30 \mathrm{AU}$ (Fig. 11d). The most massive embryos $\left(>10^{27} \mathrm{~g}\right)$ eventually migrate inward leaving only moderately large embryos in the original location of the planetesimal annulus. Actual effects of PDM on planetary accretion are under investigation adopting wide planetesimal disks (but see Figs. 15 and 16 of Morishima (2015)).

\subsection{Effects of initial mass distribution}

We have only considered planetary accretion starting with equal-mass planetesimals, except for the simulations shown in Fig. 8, It is highly likely, however, that initial planetesimals have an extended mass distribution. We discuss its effect but limit to the case where an initial planetesimal mass distribution is given by a single power-law with the exponent $q_{0}$, the upper and lower cut-off masses, $m_{0 \mathrm{U}}$ and $m_{0 \mathrm{~L}}$.

Since $q$ increases up to $\sim-2.5$ during runway growth for the case of initially equal-mass planetesimals, a similar result is expected as long as $q_{0}<-2.5$. For $q_{0}>-2.0$, on the other hand, both the mass and stirring power concentrate near $m_{0 \mathrm{UU}}$. Thus, accretion proceeds in a similar fashion to a case of initial equal-mass planetesimals with $m_{0} \sim m_{0 \mathrm{U}}$ while the initial mass slope 
for $m<m_{0 \mathrm{U}}$ is roughly retained. Indeed, these predictions are found to be true in simulations (Fig. 10). The mass distribution slope of $q \sim-2.5$ during runway growth is realized for all bodies for $q_{0}<-2.5$ whereas this slope is seen only for bodies with $m>m_{0 \mathrm{U}}$ for $q_{0}>-2.0$.

It is difficult to predict the evolution of the mass spectrum if $-2.5<q_{0}<-2.0$. For a specific case with $q_{0}=-2.3$ (Fig. 10), planetary accretion proceeds with nearly retaining a single power-law mass distribution during runway growth even for bodies more massive than $m_{0 \mathrm{U}}$. It is unclear whether a single power-law is always realized for other cases. However, we assume it for the discussion below.

The theory described in Section 2 is probably extended to the case with an initial mass distribution, as follows. If the trans-Hill stage appears, as is the case for all three simulations shown in Fig. 10, the planetary mass $M_{\text {tran }}$ at the onset of the trans-Hill stage is given by Eq. (23) but with $q$ and $m_{0}$ modified as follows:

$$
\begin{aligned}
& q=-2.5, m_{0}=m_{0 \mathrm{~L}}\left(\text { for } q_{0}<-2.5\right), \\
& q=q_{0}, m_{0}=m_{0 \mathrm{~L}}\left(\text { for }-2.5<-q_{0}<-2.0\right), \\
& q=-2.5, m_{0}=m_{0 \mathrm{U}}\left(\text { for } q_{0}>-2.0\right) .
\end{aligned}
$$

If $M_{\text {tran }}$ for $-2.5<q_{0}<-2.0$ is larger than $M_{\text {tran }}$ for $q_{0}>-2.0$, the former is probably replaced by the latter. As long as the trans-Hill stage appears, the planetary mass at the onset of oligarchic growth is given by $M_{\mathrm{oli}, \mathrm{S}}$, as repeatedly discussed. If the initial planetesimals are massive and oligarchic growth directly commences after runway growth, $M_{\mathrm{oli}, \mathrm{L}}$ is given by Eq. (13) but again $q$ and $m_{0}$ are modified as shown above. To repeat, the modification for $-2.5<q_{0}<-2.0$ is speculative and more simulations or advanced theories are necessary.

\subsection{Effects of damping forces}

Collisional damping (or fragmentation) and gas drag are known to be important during oligarchic growth (Kokubo and Ida, 2000; Chambers, 2008; Kobayashi et al., 2010). We discuss whether these effects are important already at the onset of oligarchic growth and, if so, whether the planetary mass $M_{\text {oli }}$ is modified by these effects.

\subsubsection{Collisional damping} 2014)

The velocity change rate due to collisional damping is give as (Goldreich et al., 2004; Lithwick,

$$
\left(\frac{1}{u} \frac{\mathrm{d} u}{\mathrm{~d} t}\right)_{\text {coll }}=\frac{\sigma \Omega}{\rho s} .
$$

The equilibrium velocity determined by the balance between viscous stirring of large bodies [Eq. (4)] and collisional damping is given as

$$
\left(\frac{u}{v_{\mathrm{H}}}\right)_{\text {coll }}=\left(\frac{s \Sigma}{R \sigma} \alpha^{-2}\right)^{p_{c}}=\left(\frac{\rho s R_{\mathrm{H}}}{a \sigma}\right)^{p_{c}},
$$

where the power-law index $p_{c}$ is $1 / 4$ for $u>v_{\mathrm{H}}$ and 1 for $u \leq v_{\mathrm{H}}$ and we used the equation for oligarchic growth $\Sigma a=R R_{\mathrm{H}} \rho \alpha^{2}$ [Eq. (10)] with dropping off order of unity factors in the second equality. If $\left(u / v_{\mathrm{H}}\right)_{\text {coll }}$ is lower than $\left(u / v_{\mathrm{H}}\right)_{\text {oli }}$ [Eq. (27)], collisional damping cannot be ignored at the onset of oligarchic growth or in the earlier stages. In Fig. 4, we plot $\left(u / v_{\mathrm{H}}\right)_{\text {coll }}$ derived using 
$R_{\mathrm{H}}$ for the oligarchic body with a mass of $M_{\mathrm{oli}}$ and compare it with $\left(u / v_{\mathrm{H}}\right)_{\text {oli }}$. Collisional damping is found to be important for small $s$ and $a$, or $s<R \alpha^{3 / 4}$.

Two possibilities were indicated for evolution of $\Sigma / \sigma$ during the trans-Hill stage in the collisional regime $\left(s<R \alpha^{3 / 4}\right)$. If $\left(u / v_{\mathrm{H}}\right)_{\text {coll }}$ is retained to be about unity (Lithwick, 2014), $\Sigma / \sigma=\alpha^{2} R / s$. On the other hand, if equal accretion is realized in the regime of $\alpha^{1 / 2}<u / v_{\mathrm{H}}<1$ (Shannon et al., 2016) $),\left(u / v_{\mathrm{H}}\right)_{\text {coll }}=\alpha^{-3 / 4}(s / R)^{1 / 2}$ and $\Sigma / \sigma=\alpha^{4 / 5}(R / s)^{1 / 2}$. In either case, $\Sigma / \sigma$ for the collisional regime is expected to increase with $R$ and eventually exceed that for the collisionless regime $(\Sigma / \sigma)_{\mathrm{S}}$ [Eq. (24)]. Thus, $M_{\text {oli }}$ for the collisional regime is expected to be larger as well.

Collisional damping is automatically included in all of our simulations (Section 3), and it should be particularly important for the simulation with $a=0.1 \mathrm{AU}$ and $m_{0}=10^{13} \mathrm{~g}$. In this simulation, we see that $\Sigma$ indeed increases with $R$ during the trans-Hill stage but much more gradually than the theoretical prediction (see Fig. $5 \mathrm{5}$ a for $m_{0}=10^{17} \mathrm{~g}$ as this case gives a slope similar to that for $m_{0}=10^{13} \mathrm{~g}$ ). The mass $M_{\text {oli }}$ also agrees with that for the collisionless regime rather than that for the collisional regime. The discrepancy is caused by a fact that significant depletion of the smallest bodies occurs in simulations while the theory assumes a fixed $\sigma$. As the surface density of the smallest bodies decreases, so is the the effect of collisional damping.

The effect of depletion of the smallest bodies is expected to be relatively unimportant at large $a$ or small $\alpha$ although collisional damping at large $a$ is only important for very small $s$. Shannon et al. (2016) performed simulations for formation of cold KBOs from cm-sized particles and showed that equal accretion is actually realized in the collisional case as their theory predicted.

\subsubsection{Gas drag}

The effect of gas drag is expected to play a similar role to collisional damping. The velocity change due to gas drag in the quadratic regime is given as

$$
\left(\frac{1}{u} \frac{\mathrm{d} u}{\mathrm{~d} t}\right)_{\operatorname{gas}}=\frac{\rho_{\mathrm{gas}} u}{\rho s}
$$

where $\rho_{\text {gas }}$ is the gas density. The equilibrium velocity determined by the balance between viscous stirring of large bodies and gas drag is

$$
\left(\frac{u}{v_{\mathrm{H}}}\right)_{\mathrm{gas}}=\left(\frac{s \Sigma}{R R_{\mathrm{H}} \rho_{\mathrm{gas}}} \alpha^{-2}\right)^{p_{g}}=\left(\frac{\rho s}{a \rho_{\mathrm{gas}}}\right)^{p_{g}}
$$

where the power-law index $p_{g}$ is $1 / 5$ for $u>v_{\mathrm{H}}$ and $1 / 2$ for $u \leq v_{\mathrm{H}}$ and we again used the equation

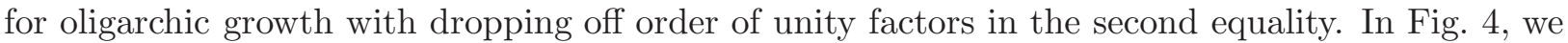
plot $\left(u / v_{\mathrm{H}}\right)_{\text {gas }}$ assuming the gas density for the MMSN $\rho_{\text {gas }}=1.4 \times 10^{-9}(a / 1 \mathrm{AU})^{-11 / 4} \mathrm{~g} \mathrm{~cm}^{-3}$. Gas drag is found to be important for small $s$ as expected and also for large $s$.

For the case of small $m_{0}$, we expect $\Sigma / \sigma \propto R^{2 / 3}$ during the trans-Hill stage, if equal accretion is realized in the regime of $\alpha^{1 / 2}<u / v_{\mathrm{H}}<1$. Here, we ignored mass depletion due to radial migration of small bodies. As well as the case with collisional damping, the expected value of $\Sigma / \sigma$ and $M_{\text {oli }}$ are larger than those for the non-damping case.

The condition for $\left(u / v_{\mathrm{H}}\right)_{\text {gas }}<\left(u / v_{\mathrm{H}}\right)_{\text {oli }}$ is also written as $\mathrm{St}<\alpha^{-1 /\left(8 p_{g}\right)} / e(\sim 1000$ at the onset of oligarchic growth), where St $\simeq \rho s /\left(\rho_{\text {gas }} a e\right)$ is the Stokes number for the quadratic gas drag regime. For St $<100$, the accretion rate of a large body we adopted [Eq. (7)] is no longer valid since small bodies' orbits in the Hill sphere of a large body are highly modified by gas drag. The planetary accretion in this regime, called pebble accretion, is generally very efficient (Ormel and Klahr, 
2010; Lambrechts and Johansen, 2012; Morbidelli and Nesvornv́, 2012). This effect was successfully studied for formation of giant planets (Lambrechts and Johansen, 2014; Morbidelli et al., 2015; Levison et al., 2015a) and terrestrial planets (Levison et al., 2015b; Sato et al., 2016). This mechanism is also able to reproduce the mass distribution of asteroids and potentially that for KBOs (Johansen et al., 2015a,b).

For the case of large $m_{0}\left(>m_{0, \text { crit }}\right)$, to derive $M_{\text {oli, }, ~}$, we assumed a single power-law for the mass distribution. The power-law index $q$ always takes $\simeq-2.5$ whether gas drag is included or not (Kokubo and Ida, 2000; Morishima et al., 2013). Thus, $M_{\text {oli, L }}$ is probably not modified by gas drag, although it can accelerate planetary accretion.

\section{Summary}

Oligarchic growth commences when the velocity dispersion $u$ relative to the Hill velocity $v_{\mathrm{H}}$ of the largest body takes its minimum. We found that if the initial planetesimal mass $m_{0}$ is small enough, $u / v_{\mathrm{H}}$ becomes as low as unity during the intermediate (trans-Hill) stage between the runaway and oligarchic growth stages. In this case, $M_{\text {oli }}$ is independent of $m_{0}$. If $m_{0}$ is large, on the other hand, oligarchic growth commences directly after runaway growth, and $M_{\text {oli }} \propto m_{0}^{3 / 7}$. We found that the contribution of mutual merging between large bodies to their growth is comparable to or slightly larger than that due to merging with small planetesimals at any stage of planetary accretion.

The planetary mass $M_{\text {oli }}$ for the solid surface density of the Minimum Mass Solar Nebula is close to the masses of the dwarf planets in a reasonable range of $m_{0}$. This indicates that they are likely to be the largest remnant planetesimals that failed to become planets. Bodies more massive than $M_{\text {oli }}$ probably merged with massive protoplanets once existed. For KBOs, alternatively, their accretion might have been externally halted by orbital instability of giant planets when the largest KBOs reached $M_{\text {oli }}$ in mass.

The mass distribution bump at $10^{21} \mathrm{~g}(s \sim 50 \mathrm{~km})$ and the slope, $q \simeq-2.7$, of hot KBOs are reproduced if $m_{0}$ is the bump mass. On the other hand, the small initial planetesimals, $m_{0} \sim 10^{13}$ $\mathrm{g}$ or less, are favored for reproducing the slope of asteroids, $q \simeq-2.0$, while the bump at $10^{21} \mathrm{~g}$ can be reproduced by introducing a small number of asteroid seeds each with mass of $10^{19} \mathrm{~g}(10$ $\mathrm{km}$ in radius). The present study rather focused on the basic theory that connects between the initial and the final mass distributions. Detailed comparison between modeled and observed mass distributions should be made somewhere else.

\section{Acknowledgements}

We thank the reviewers, Yoram Lithwick and Eiichiro Kokubo, for their constructive comments that helped us to improve the manuscript. This research was carried out in part at the Jet Propulsion Laboratory, California Institute of Technology, under contract with NASA. Government sponsorship acknowledged. Simulations were performed using a JPL supercomputer, Aurora.

\section{References}

Adams, E., Gulbis, A., Elliot, J., Benecchi, S., Buie, M., Trilling, D., Wasserman, L., 2014. De-biased populations of kuiper belt objects from the deep ecliptic survey. Astron. J. 148, 55.

Barnes, R., Quinn, T., Lissauer, J., Richardson, D., 2009. N-body simulations of growth of $1 \mathrm{~km}$ planetesimals at 0.4 au. Icarus 203, 626-643. 
Batygin, K., Brown, M., Fraser, W., 2011. Retention of a primordial cold classical kuiper belt in an instability-driven model of solar system formation. Astrophys. J. 738, 13.

Benz, W., Asphaug, E., 1999. Catastrophic disruption revisited. Icarus 142, 5-20.

Bernstein, G., Trilling, D., Allen, R., Brown, M., Holman, M., Malhotra, R., 2004. The size distribution of transneptunian bodies. Astron. J. 128, 1364-1390.

Bottke, W., Durda, D., Nesvorný, D., Jedicke, R., Morbidelli, A., Vokrouhlický, D., Levison, H., 2005. The fossilized size distribution of the main asteroid belt. Icarus 175, 111-140.

Bromley, B., Kenyon, S., 2011. Migration of planets embedded in a circumstellar disk. Astrophys. J. 735, 29.

Brown, M., 2013. On the size, shape, and density of dwarf planet makemake. Astrophys. J. 767, L7.

Brown, M., Schaller, E., 2007. The mass of dwarf planet eris. Science 316, 1585.

Campo Bagatin, A., Benavidez, P., 2012. Collisional evolution of trans-neptunian object populations in a nice model environment. Mon. Not. R. Astron. Soc. 423, 1254-1266.

Chambers, J., 2006. A semi-analytic model for oligarchic growth. Icarus 180, 496-513.

Chambers, J., 2008. Oligarchic growth with migration and fragmentation. Icarus 198, 256-273.

Chambers, J., 2010. Planetesimal formation by turbulent concentration. Icarus 208, 505-517.

Cuzzi, J., Hogan, R., Bottke, W., 2010. Towards initial mass function for asteroids and kuiper belt objects. Icarus 208, 518-538.

Cuzzi, J., Hogan, R., Shariff, K., 2008. Toward planetesimals: Dense chondrule clumps in the protoplanetary nebula. Astrophys. J. 687, 1432-1447.

Duncan, M., Levison, H., Lee, M., 1998. A multiple time step symplectic algorithm for integrating close encounters. Astron. J. 116, 2067-2077.

Fraser, W., Brown, M., Morbidelli, A., Parker, A., Batygin, K., 2014. The absolute magnitude distribution function of kuiper belt objects. Astrophys. J. 782, 100.

Fraser, W., Brown, M., Schwamb, M., 2010. The luminosity function of the hot and cold kuiper belt populations. Icarus 210, 944-955.

Garaud, P., Meru, F., Galvagni, M., Olczak, C., 2013. From dust to planetesimals: an improved model for collisional growth in protoplanetary disks. Astrophy. J. 764, 146.

Genda, H., Kokubo, E., Ida, S., 2012. Merging criteria for giant impacts of protoplanets. Astrophys. J. $744,137$.

Goldreich, P., Lithwick, Y., Sari, R., 2004. Planet formation by coagulation: A focus on uranus and neptune. Ann. Rev. Astron. Astrophys. 42, 549-601.

Gomes, R., Levison, H., Tsiganis, K., Morbidelli, A., 2005. Origin of the cataclysmic late heavy bombardment period of the terrestrial planets. Nature 436, 466-469.

Greenberg, R., Wacker, J., Hartmann, W., Chapman, C., 1978. Planetesimals to planets: Numerical simulation of collisional evolution. Icarus 35, 1-26.

Greenzweig, Y., Lissauer, J., 1990. Accretion rates of protoplanets. Icarus 87, 40-77.

Hahn, J., Malhotra, R., 2005. Neptune's migration into a stirred-up kuiper belt: A detailed comparison of simulations to observations. Astron. J. 130, 2392-2414.

Hayashi, C., 1981. Structure of the solar nebula, growth and decay of magnetic fields and effects of magnetic and turbulent viscosities on the nebula. Suppl. Prog. Theoret. Phys. 70, 35-53.

Hopkins, P., 2016. Jumping the gap: the formation conditions and mass function of 'pebble-pile' planetesimals. Mon. Not. R. Astron. Soc. 456, 2383-2405.

Ida, S., 1990. Stirring and dynamical friction rates of planetesimals in the solar gravitational field. Icarus 88, $129-145$.

Ida, S., Bryden, G., Lin, D., Tanaka, H., 2000. Orbital migration of neptune and orbital distribution of trans-neptune objects. Astrophys. J. 534, 428-445.

Ida, S., Makino, J., 1993. Scattering of planetesimals by a protoplanet: slowing down of runaway growth. Icarus 106, 210-227.

Ida, S., Nakazawa, K., 1989. Collisional probability of planetesimals revolving in the solar gravitational field. iii. Astron. Astrophys. 224, 303-315.

Jedicke, R., Larsen, J., Spahr, T., 2002. Observational selection effects in asteroid surveys., in: Bottke, W., Cellino, A., Policchi, P., Binzel, R. (Eds.), Asteroids III. University of Arizona Press, Tucson, pp. 71-87.

Johansen, A., Jacquet, E., Cuzzi, J., Morbidelli, A., Gounelle, M., 2015a. New paradigms for asteroid formation., in: Michel, P., DeMeo, F., Bottke, W. (Eds.), Asteroids IV. University of Arizona Press, Tucson, pp. 471-492.

Johansen, A., Mac Low, M.M., Lacerda, P., Bizzarro, M., 2015b. Growth of asteroids, planetary embryos, and kuiper belt objects by chondrule accretion. Science Advances 1, 1500109.

Johansen, A., Oishi, J., Mac Low, M.M., Klahr, H., Henning, T., Youdin, A., 2007. Rapid planetesimal formation in turbulent circumstellar disks. Nature 448, 1022-1025. 
Kataoka, A., Tanaka, H., Okuzumi, S., Wada, K., 2013. Fluffy dust forms icy planetesimals by static compression. Astron. Astrophys. 557, L4.

Kenyon, S., Bromley, B., 2012. Coagulation calculations of icy planet formation at 15-150 au: A correlation between the maximum radius and the slope of the size distribution for trans-neptunian objects. Astron. J. 143, 63.

Kirsh, D., Duncan, M., Brasser, R., Levison, H., 2009. Simulations of planet migration driven by planetesimal scattering. Icarus 199, 197-209.

Kobayashi, H., Tanaka, H., Krivov, A., Inaba, S., 2010. Planetary growth with collisional fragmentation and gas drag. Icarus 209, 836-847.

Kokubo, E., Genda, H., 2010. Formation of terrestrial planets from protoplanets under a realistic accretion condition. Astrophys. J. 714, L21-L25.

Kokubo, E., Ida, S., 1996. On runaway growth of planetesimals. Icarus 123, 180-191.

Kokubo, E., Ida, S., 1998. Oligarchic growth of protoplanets. Icarus 131, 171-178.

Kokubo, E., Ida, S., 2000. Formation of protoplanets from planetesimals in the solar nebula. Icarus 143, 15-27.

Kokubo, E., Ida, S., 2002. Formation of protoplanet systems and diversity of planetary systems. Astrophys. J. 581, 666-680.

Kominami, J., Daisaka, H., Makino, J., Fujimoto, M., 2016. Global high-resolution N-body simulation of planet formation i: Planetesimal driven migration. Astrophys. J. 819, 30.

Lambrechts, M., Johansen, A., 2012. Rapid growth of giant-cores by pebble accretion. Astron. Astrophys. 544 , A32.

Lambrechts, M., Johansen, A., 2014. Forming the cores of giant planets from the radial pebble flux in protoplanetary disks. Astron. Astrophys. 572, A107.

Leinhardt, Z., Stewart, S., 2012. Collisions between gravity-dominated bodies. i. outcome regimes and scaling laws. Astrophys. J. 745, 79.

Levison, H., Duncan, M., Thommes, E., 2012. A lagrangian integrator for planetary accretion and dynamics. Astrophys. J. 144, 119.

Levison, H., Kretke, K., Duncan, M., 2015a. Growing the gas-giant planets by the gradual accumulation of pebbles. Nature 524, 322-324.

Levison, H., Kretke, K., Walsh, K., Bottke, W., 2015b. Growing the terrestrial planets from the gradual accumulation of sub-meter sized objects. Proc. Natl. Acad. Sci. 112, 14180-14185.

Levison, H., Morbidelli, A., VanLaerhoven, C., Gomes, R., Tsiganis, K., 2008. Origin of the structure of the kuiper belt during a dynamical instability in the orbits of uranus and neptune. Icarus 196, 258-273.

Levison, H., Thommes, E., Duncan, M., 2010. Modeling the formation of giant planet cores. i. evaluating key processes. Astron. J. 139, 1297-1314.

Lithwick, Y., 2014. After runaway: The trans-hill stage of planetesimals growth. Astrophys. J. 780, 22.

Lykawka, P., Mukai, T., 2008. An outer planet beyond pluto and the origin of trans-neptunian belt architecture. Astron. J. 135, 1161-1200.

Makino, J., Fukushige, T., Funato, Y., Kokubo, E., 1998. On the mass distribution of planetesimals in the early runway stage. New Astron. 3, 411-417.

Malhotra, R., 1993. The origin of pluto's peculiar orbit. Nature 365, 819-821.

Minton, D., Levison, H., 2014. Planetesimal-driven migration of terrestrial planet embryos. Icarus 232, $118-132$.

Morbidelli, A., Bottke, W., Nesvorný, D., Levison, H., 2009. Asteroids were born big. Icarus 204, 558-573.

Morbidelli, A., Lambrechts, M., Jacobson, S., Bitsch, B., 2015. The great dichotomy of the solar system: Small terrestrial embryos and massive giant planet cores. Icarus 258, 418-429.

Morbidelli, A., Levison, H., Tsiganis, K., Gomes, R., 2005. Chaotic capture of jupiter's trojan asteroids in the early solar system. Nature 435, 462-465.

Morbidelli, A., Nesvorný, D., 2012. Dynamics of pebbles in the vicinity of a growing planetary embryo: hydrodynamical simulations. Astron. Astrophys. 546, A18.

Morishima, R., 2015. A particle-based hybrid code for planet formation. Icarus 260, 368-395.

Morishima, R., Golabek, G., Samuel, H., 2013. N-body simulations of oligarchic growth of mars: Implications for hf-w chronology. Earth Planet. Sci. Lett. 366, 6-16.

Morishima, R., Schmidt, M., Stadel, J., Moore, B., 2008. Formation and accretion history of terrestrial planets from runaway growth through to late time: Implications for orbital eccentricity. Astrophys. J. 685, 1247-1261.

Morishima, R., Stadel, J., Moore, B., 2010. From planetesimals to terrestrial planets: $N$-body simulations including the effects of nebular gas and giant planets. Icarus 207, 517-535.

Nesvorný, D., Morbidelli, A., 2012. Statistical study of the early solar system's instability with four, five, and six giant planets. Astron. J. 144, 117.

Okuzumi, S., Tanaka, H., Kobayashi, H., Wada, K., 2012. Rapid coagulation of porous dust aggregates outside the 
snow line: A pathway to successful icy planetesimal formation. Astrophys. J. 752, 106.

Ormel, C., Dullemond, C., Spaans, M., 2010a. Accretion among preplanetary bodies: The many faces of runaway growth. Icarus 210, 507-538.

Ormel, C., Dullemond, C., Spaans, M., 2010b. A new condition for the transition from runaway to oligarchic growth. Astrophys. J. Lett. 714, L103-L107.

Ormel, C., Ida, S., Tanaka, H., 2012. Migration rates of planets due to scattering of planetesimals. Astrophys. J. 758,80 .

Ormel, C., Klahr, H., 2010. The effect of gas drag on the growth of protoplanets: Analytic expressions for the accretion of small bodies in laminar disks. Astron. Astrophys. 520, A43.

Parker, A., Kavelaars, J., 2012. Collisional evolution of ultra-wide trans-neptunian binaries. Astrophys. J. 744 , 139.

Petit, J.M., et al., 2011. The canada-france ecliptic plane survey - full data release: The orbital structure of the kuiper belt. Astron. J. 142, 131.

Rafikov, R., 2003. Dynamical evolution of planetesimals in protoplanetary disks. Astron. J. 126, 2529-2548.

Ragozzine, D., Brown, M., 2009. Orbits and masses of the satellites of the dwarf planet haumea (2003 el61). Astron. J. 137, 4766-4776.

Richardson, D., Quinn, T., Stadel, J., Lake, G., 2000. Direct large-scale N-body simulations of planetesimal dynamics. Icarus $143,45-49$.

Russell, C., et al., 2012. Dawn at vesta: Testing the protoplanetary paradigm. Sceince 336, 684-686.

Russell, C., et al., 2015. Dawn arrives at ceres: Results of the survey orbit. EPSC abstract 10, 14.

Sato, T., Okuzumi, S., Ida, S., 2016. On the water delivery to terrestrial embryos by ice pebble accretion. Astron. Astrophys. 589, A15.

Schlichting, H., Fuentes, C., Trilling, D., 2013. Initial planetesimal sizes and the size distribution of small kuiper belt objects. Astron. J. 146, 36.

Schlichting, H., Sari, R., 2011. Runaway growth during planet formation: Explaining the size distribution of large kuiper belt objects. Astrophys. J. 728, 68.

Shannon, A., Wu, Y., Lithwick, Y., 2015. Conglomeration of kilometer-sized planetesimals. Astrophys. J. 801, 15.

Shannon, A., Wu, Y., Lithwick, Y., 2016. Forming the cold classical kuiper belt in a light disk. Astrophys. J. 818, 175.

Stadel, J., 2001. Cosmological $N$-body simulations and their analysis. Ph.D. Thesis, Univ. of Washington.

Stern, S., et al., 2015. The pluto system: Initial results from its exploration by new horizons. Science 350, 1815.

Thomas, P., Parker, J., McFadden, L., Russell, C., Stern, S., Sykes, M., Young, E., 2005. Differentiation of the asteroid ceres as revealed by its shape. Nature 437, 224-226.

Weidenschilling, S., 2011. Initial sizes of planetesimals and accretion of the asteroids. Icarus 214, 671-684.

Wetherill, G., Stewart, G., 1989. Accumulation of a swarm of small planetesimals. Icarus 77, 330-357.

Windmark, F., Birnstiel, T., Güttler, C., Blum, J., Dullemond, C., Henning, T., 2012. Planetesimal formation by sweep-up: How the bouncing barrier can be beneficial to growth. Astron. Astrophys. 540, A73. 